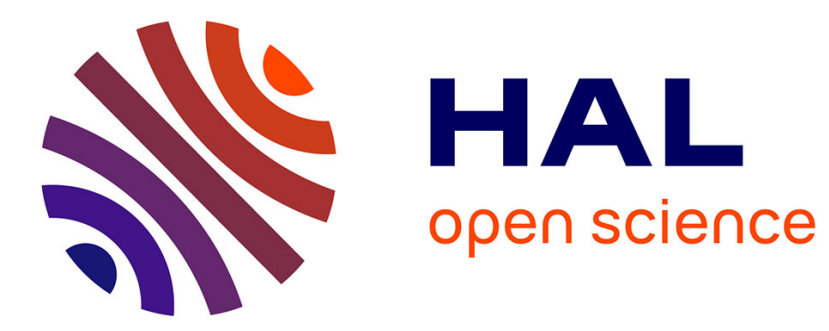

\title{
Multifluid Flows: A Kinetic Approach
}

Florent Berthelin, Thierry Goudon, Sebastian Minjeaud

\section{To cite this version:}

Florent Berthelin, Thierry Goudon, Sebastian Minjeaud. Multifluid Flows: A Kinetic Approach. Journal of Scientific Computing, 2016, 66 (2), pp.792-824. 10.1007/s10915-015-0044-1 . hal-01158016

\section{HAL Id: hal-01158016 https://hal.science/hal-01158016}

Submitted on 29 May 2015

HAL is a multi-disciplinary open access archive for the deposit and dissemination of scientific research documents, whether they are published or not. The documents may come from teaching and research institutions in France or abroad, or from public or private research centers.
L'archive ouverte pluridisciplinaire HAL, est destinée au dépôt et à la diffusion de documents scientifiques de niveau recherche, publiés ou non, émanant des établissements d'enseignement et de recherche français ou étrangers, des laboratoires publics ou privés. 


\title{
Multifluid flows: a kinetic approach
}

\author{
F. Berthelin*1,3 , T. Goudon ${ }^{\dagger 1,3}$, and S. Minjeaud ${ }^{\ddagger 2,3}$ \\ ${ }^{1}$ Project COFFEE, INRIA Sophia Antipolis Méditerranée \\ ${ }^{2}$ Project CASTOR, INRIA Sophia Antipolis Méditerranée \\ ${ }^{3}$ Labo J.-A. Dieudonné, UMR 7351 CNRS-Univ. Nice Sophia Antipolis, \\ Parc Valrose, F-06108 Nice, France
}

May 28, 2015

\begin{abstract}
We propose a numerical scheme for the simulation of fluid-particles flows with two incompressible phases. The numerical strategy is based on a finite volume discretization on staggered grids, with a flavor of kinetic schemes in the definition of the numerical fluxes. We particularly pay attention to the difficulties related to the volume conservation constraint and to the presence of a close-packing term which imposes a threshold on the volume fraction of the disperse phase. We are able to identify stability conditions on the time step to preserve this threshold and the energy dissipation of the original model. The numerical scheme is validated with the simulation of sedimentation flows. Keywords. Multi-phase flows. Kinetic schemes. Close-packing. Incompressible flows.
\end{abstract}

\section{Introduction}

This work is concerned with non linear systems of PDEs intended to describe complex mixture flows, referred to as "multifluid" flows, where a relatively disperse phase interacts with a carrier fluid. Such problems arise in the modeling of many industrial processes or natural phenomena. A typical example relies on the conception of fluidized beds. In such devices, particles become suspended in the fluid stream, which, in turn, promotes an intimate contact between the particles and the fluid. This technique is used, for instance, to facilitate the combustion of material with a low caloric content, the absorption of the pollutants deriving from the combustion, the heat exchanges in

\footnotetext{
*Florent. Berthelin@unice.fr

$\dagger$ thierry.goudon@inria.fr

${ }_{\ddagger}^{\ddagger}$ minjeaud@unice.fr
} 
peb ble bed reactors, etc. Therefore, the fluidized bed consists of a complex fluid-solid mixture that exhibits fluid-like properties, with substancial density inhomogeneities.

A numerical challenge consists in reproducing density contrasts and transition regions, which in turn, govern the overall features of the flow. Of course, one is interested in the behavior of macroscopic quantities, directly accessible to measurements, like density, velocity, pressure..., but we shall see that adopting a more microscopic viewpoint can be useful. Specific difficulties, that make the problem much harder than for single-phase flows can be listed as follows:

- We shall see that many aspects of the modeling might look questionable, as pointed out in [39]. While The formulation of a satisfactory set of averagedequations models emerges as the single highest priority in the modeling of complex multiphase flows we wish to adopt the following recommandation: It seems justified to take a pragmatic view of the situation, using the available models and methods with open eyes and full awareness of their potential limitations. An exploration of the sensitivity of the results to the constitutive relations, parameter values, and numerics [...] becomes a vital necessity in this field [39]. Many models proposed for such multiphase flows, which look derived on reasonable bases, exhibit non realistic instabilities, due to a lack of mathematical structure (reduced region of hyperbolicity).

- Anyway, we face strongly nonlinear coupling of the equations describing the interacting phases.

- The structure of the equations is somehow unusual, involving naturally nonconservative terms, especially with pressure terms.

- The problems is naturally multi-scale, and the stiffness of source terms is a source of numerical difficulties.

- Finally, it is a challenge to design a numerical method which can preserve the natural properties of the model, like positivity, volume and mass conservation, energy/entropy dissipation...

In what follows we shall consider a purely hydrodynamic model, see (15)-(19) below, where the disperse and the carrier phases are described by their respective mass density and velocity, and a common pressure field. We shall assume the incompressibility of the two phases. It leads to the constraint that the mean volume velocity of the flow is solenoidal, which, in turn, defines the pressure. Furthermore, we will pay a specific attention to the so-called "close-packing" effects which are intended to maintain the particle volume fraction below a certain threshold. We start by discussing the modeling issues in Section 2, on the basis of the Eulerian-Lagrangian framework where the disperse phase is described by means of the distribution function of particles in phase space. The hydrodynamic model we wish to investigate is just obtained as a mono-kinetic version of this microscopic model, see Section 2.2. This derivation allows us to bring out remarkable dissipation properties of the model. We discuss the conception of specific numerical strategies to solve this PDEs system in Section 3. In particular we pay attention to the following two difficulties. Firstly, the close-packing corresponds to pressure forces on the particles which become singular as the particle 
volume fraction approaches the threshold. The numerical scheme should preserve the corresponding bound on the volume fraction. Secondly, the behavior of the two phases is coupled by the drag forces and the implicit definition of the pressure. Hence, the volume conservation requires a careful treatment at the discrete level. For the coupling, our approach remains in the spirit of projection methods used for incompressible fluids. However, it needs a suitable adaptation, that takes into account the definition of the discrete convection fluxes. We adopt the Finite Volume framework and the microscopic viewpoint is used to design numerical fluxes. We propose a specific "kinetic scheme", adapted from [6], dedicated to handle the close-packing terms and the volume conservation constraint. The method relies on the following two key ingredients:

- Due to the complexity of the close-packing term, which takes the form of a pressure force on the disperse phase, the definition of the equilibrium states for the kinetic scheme is quite unusual. In turn, convection terms and pressure terms are approximated in a different way (roughly speaking, the former are upwinded and the latter are centered).

- We find convenient to work on staggered grids where densities, pressures and velocities are approached on different cells. This has two consequences. First of all the scheme derived that way can be shown to be energy decaying (despite the complexity of the pressure law which seems to exclude the identification of the equilibrium states by means of a minimization problem, see Remark 3.2). Second of all, this strategy yields a relevant way to take into account at the discrete level the constraint in order to conserve exactly the total volume.

Our analysis shows that the respect of the close-packing threshold induces delicate stability constraints. We conclude by offering a series of numerical tests in Section 4.

\section{Modeling Issues}

We refer the reader to $[22,38,39]$ for a thorough introduction to mathematical models for mixture flows. As said above, many aspects in the derivation of the equations remain under debate. Our viewpoint is to go back to a more microscopic description of the fluid-particle interaction. Of course, the modeling basis of the corresponding coupled hydro-kinetic systems are still questionable. But, we expect to be able to set up hierarchies of models and to identify how some remarkable properties (like energy or entropy dissipation) are transmitted throughout the hierarchy. This viewpoint might also help in designing numerical schemes.

\subsection{Kinetic-Fluid Models}

As a starting point we adopt a hybrid description of the flow: the carrier phase is a fluid described by using concepts of continuum mechanics, but for the particles, we work with the viewpoint of statistical physics. This is the so-called Eulerian-Lagrangian framework which leads to couple hydrodynamic systems and kinetic equations. Similar models are used for the simulation of particle-laden flows in $[1,2,33,34,35,41]$. For 
the simpler case of thin sprays [33], asymptotic issues are discussed e. g. in [10, 23, 24], and schemes dedicated to these regimes are presented in $[11,25,26,27]$.

\subsubsection{Description of the fluid phase}

The fluid is described by the mass density $\varrho_{f}$, the velocity $u_{f}$ and the temperature $T$ which depend on the time $t \geq 0$ and space variable $x \in \Omega \subset \mathbf{R}^{N}, N=3$. We also need the volume fraction $\alpha_{f}$ occupied by the fluid. The evolution of these quantities is governed by the mass conservation

$$
\partial_{t}\left(\alpha_{f} \varrho_{f}\right)+\nabla_{x} \cdot\left(\alpha_{f} \varrho_{f} u_{f}\right)=0
$$

and the momentum equation

$$
\partial_{t}\left(\alpha_{f} \varrho_{f} u_{f}\right)+\nabla_{x} \cdot\left(\alpha_{f} \varrho_{f} u_{f} \otimes u_{f}\right)+\alpha_{f} \nabla_{x} P_{f}=\nabla_{x} \cdot\left(\alpha_{f} \tau\right)+\mathbf{F}_{f p}+\alpha_{f} \varrho_{f} g
$$

where $\tau$ is the viscous stress tensor, $g$ is the gravity acceleration while $\mathbf{F}_{f p}$, which will be detailed below (see section 2.1.3), contains momentum exchanges with the disperse phase. The viscous stress tensor is defined by

$$
\tau=\mu\left(\nabla_{x} u_{f}+\left(\nabla_{x} u_{f}\right)^{\top}-\frac{2}{3}\left(\nabla_{x} \cdot u_{f}\right) \mathrm{Id}\right)
$$

where the constant $\mu>0$ stands for the dynamic viscosity of the fluid and $\left(\nabla_{x} u_{f}\right)^{\top}$ stands for the transpose of the matrix $\nabla_{x} u_{f}$.

We assume that the fluid flow is homogeneous, isothermal and incompressible, so that

$$
\varrho_{f}(t, x)=\varrho_{f}>0 \text { is constant, }
$$

and the temperature is constant as well. Therefore inhomogeneities are entirely described by the volume fraction $\alpha_{f}(t, x)$. The definition of the pressure $P_{f}$ is related to the modeling assumptions both on the carrier and on the dispersed phases, and it will be discussed below (see section 2.1.3).

\subsubsection{Description of the dispersed phase}

We turn to the description of the dispersed phase. We restrict the discussion to a single-specie of particles, characterized by the mass density $\varrho_{p}>0$. We assume that particles are homogeneous spheres, with radius $a>0$. Let $F(t, x, \xi, a)$ stand for the particle distribution function: the integral

$$
\iiint_{A \times U \times O} F(t, x, \xi, a) \mathrm{d} a \mathrm{~d} \xi \mathrm{d} x
$$

gives the probable number of particles per volume unit which, at time $t \geq 0$, lies in the space domain $O \subset \Omega \subset \mathbf{R}^{N}$, with a velocity belonging to $U \subset \mathbf{R}^{N}$, and a radius in the set $A \subset[0, \infty)$. The quantity

$$
\alpha_{p}(t, x)=\iint_{[0, \infty) \times R^{3}} F(t, x, \xi, a) \frac{4}{3} \pi a^{3} \mathrm{~d} a \mathrm{~d} \xi
$$


is thus the volume fraction locally occupied by the particles. Neglecting size-variations (that are due to coagulation and break-up phenomena), the particle distribution function obeys the following Vlasov-type equation

$$
\partial_{t} F+\xi \cdot \nabla_{x} F+\nabla_{\xi} \cdot(A F)=0 .
$$

The acceleration term $A(t, x, \xi, a)$ can be split in two parts as follows

$$
\varrho_{p} A(t, x, \xi, a)=\mathbf{F}_{p}(t, x, \xi, a)+\mathbf{F}_{p f}(t, x, \xi, a) .
$$

The term $\mathbf{F}_{p f}$ is intended to account for the coupling forces exerted by the fluid on particles while $\mathbf{F}_{p}$ accounts for the following physical phenomena:

a) Pressure gradient term $-\nabla_{x} P_{p}$.

b) External forces, that can be of very different nature: gravity, centrifugal forces, electromagnetic forces... Restricting to gravity driven flows, the force $\varrho_{p} g$ is proportional to the gravity acceleration $g$.

c) Equation (2) does not contain Boltzmann-like collision terms. However collision frequency becomes high when the particle volume fraction increases. Then interparticles stresses are modeled by an additional force term that reads

$$
-\frac{1}{\alpha_{p}} \nabla_{x}\left(\mathrm{P}_{\star} \pi\left(\alpha_{p}\right)\right) .
$$

Here, $\mathrm{P}_{\star}>0$ has the homogeneity of a pressure, and, given $0<\alpha_{\star}<1$, the so-called close-packing volume fraction $\pi:\left[0, \alpha_{\star}\right) \rightarrow[0, \infty)$ is required to satisfy

(i) $\pi$ is a function of class $\mathscr{C}^{2}$ s. t. $\pi(0)=0, \pi^{\prime}(0)=0, \lim _{\alpha_{\star}} \pi=+\infty$,

(ii) $\pi^{\prime}(\alpha)>0, \forall \alpha \in\left(0, \alpha_{\star}\right)$, and $\lim _{\alpha \rightarrow \alpha_{\star}} \int_{M}^{\alpha} \frac{1}{z} \sqrt{\pi^{\prime}(z)} \mathrm{d} z=+\infty, \forall M>0$,

(iii) $2 \pi^{\prime}(\alpha)+\alpha \pi^{\prime \prime}(\alpha) \geq 0, \forall \alpha \in\left[0, \alpha_{\star}\right)$.

This term is intended to prevent the particle volume fraction to exceed the threshold $\alpha_{\star}$. Of course, the expression of $\pi(\alpha)$ is mainly of empirical nature. The function

$$
\pi(\alpha)=\frac{\alpha^{\beta}}{\alpha_{\star}-\alpha}, \quad \beta>1
$$

fulfils (4), where, in practice, the exponent $\beta>1$ and the reference pressure $\mathrm{P}_{\star}$ are determined empirically. We refer the reader to $[1,2,3,7,29,35,34,41]$ for discussions on such pressure laws and relevant examples of applications. We will particularly focus on the treatment and the influence of this close-packing force.

Accordingly, the acceleration term $\mathbf{F}_{p}$ in (3) reads

$$
\mathbf{F}_{p}(t, x, \xi, a)=\varrho_{p} g-\nabla P_{p}-\frac{\mathrm{P}_{\star}}{\alpha_{p}} \nabla_{x} \pi\left(\alpha_{p}\right) .
$$




\subsubsection{Coupling terms}

We now turn to the description of $\mathbf{F}_{p f}$ which accounts for the forces exerted by the fluid on the particles. We only take into accounts the drag force which is proportional to the relative velocity between the particle and the surrounding fluid

$$
\mathbf{F}_{p f}=\varrho_{p} D_{p}\left(u_{f}-\xi\right)
$$

where $(t, x) \mapsto u_{f}(t, x)$ is the fluid velocity field. The drag coefficient $D_{p} \geq 0$ might have a very complicated form. It usually depends on the particulate Reynolds number

$$
\operatorname{Re}=2 \varrho_{f} \frac{\left|u_{f}-\xi\right| a}{\mu} .
$$

Examples of relevant expressions, mostly of empirical nature, can be found e.g. in $[7,18,33,35]$. As Re becomes small, the drag force depends linearly on the relative velocity $u_{f}-\xi$ (Stokes law) with

$$
D_{p}=\frac{9}{2} \frac{\mu}{\varrho_{p} a^{2}},
$$

which appears as the inverse of the Stokes settling time. In what follows, we will mainly restrict to this specific case. Besides, we have

$$
\mathbf{F}_{f p}(t, x)=-\int \mathbf{F}_{p f}(t, x, \xi, a) F(t, x, \xi, a) \frac{4}{3} \pi a^{3} \mathrm{~d} \xi \mathrm{d} a .
$$

Furthermore, the volume fractions are constrained by the relation

$$
\alpha_{p}+\alpha_{f}=1
$$

Finally, we assume that there is no pressure imbalance between the two phases, that is $P_{f}=P_{p}$ and we denote the unique pressure by $P$. This unknown is associated to the constraint (6).

\subsubsection{Recap}

For the sake of simplicity, we assume that all particles have the same radius $\bar{a}>0, F$ degenerates to a Dirac mass and we get

$$
F(t, x, \xi, a)=f(t, x, \xi) \delta(a=\bar{a}), \quad \alpha_{p}(t, x)=\frac{4}{3} \pi \bar{a}^{3} \int f(t, x, \xi) \mathrm{d} \xi .
$$


The particulate flow under consideration is described by the following fluid-kinetic model

$$
\begin{aligned}
& \partial_{t} \alpha_{f}+\nabla_{x} \cdot\left(\alpha_{f} u_{f}\right)=0, \\
& \varrho_{f}\left[\partial_{t}\left(\alpha_{f} u_{f}\right)+\nabla_{x} \cdot\left(\alpha_{f} u_{f} \otimes u_{f}\right)\right]+\alpha_{f} \nabla_{x} P-\nabla_{x} \cdot\left(\alpha_{f} \tau\right) \\
& =\alpha_{f} \varrho_{f} g+\frac{4}{3} \pi \bar{a}^{3} \int f \varrho_{p} D_{p}\left(\xi-u_{f}\right) \mathrm{d} \xi, \\
& \varrho_{p}\left[\partial_{t} f+\xi \cdot \nabla_{x} f\right]+\nabla_{\xi} \cdot\left(\left[\varrho_{p} D_{p}\left(u_{f}-\xi\right)-\nabla_{x} P-\frac{\mathrm{P}_{\star}}{\alpha_{p}} \nabla_{x} \pi\left(\alpha_{p}\right)+\varrho_{p} g\right] f\right)=0, \\
& \alpha_{f}=1-\alpha_{p}=1-\frac{4}{3} \pi \bar{a}^{3} \int f \mathrm{~d} \xi .
\end{aligned}
$$

We set

$$
q_{p}(t, x)=\alpha_{p} u_{p}(t, x)=\frac{4}{3} \pi \bar{a}^{3} \int \xi f(t, x, \xi) \mathrm{d} \xi
$$

where $u_{p}$ can be interpreted as the bulk velocity of the particles. Let us integrate (9) with respect to the velocity variable. Assuming that $f$ vanishes for large $|\xi|$, we obtain the following moment equations

$$
\partial_{t} \alpha_{p}+\nabla_{x} \cdot\left(\alpha_{p} u_{p}\right)=0
$$

and

$$
\varrho_{p}\left[\partial_{t}\left(\alpha_{p} u_{p}\right)+\nabla_{x} \cdot \mathbb{P}\right]+\alpha_{p} \nabla_{x} P+\mathrm{P}_{\star} \nabla_{x} \pi\left(\alpha_{p}\right)=\alpha_{p} \varrho_{p} g-\alpha_{p} \varrho_{p} D_{p}\left(u_{p}-u_{f}\right)
$$

where the last terms is exactly the opposite of the last term in (8), and

$$
\mathbb{P}(t, x)=\frac{4}{3} \pi \bar{a}^{3} \int \xi \otimes \xi f(t, x, \xi) \mathrm{d} \xi
$$

Hence Equations (11) and (12) do not provide a closed system because, in general, $\mathbb{P}$ is not defined by means of $\alpha_{p}$ and $q_{p}=\alpha_{p} u_{p}$. Nevertheless, the moment equations can be used to establish the following worthwhile remarks.

- Firstly, adding (7) and (11), we obtain

$$
\partial_{t}\left(\alpha_{p}+\alpha_{f}\right)=\partial_{t}(1)=0=-\nabla_{x} \cdot\left(\alpha_{f} u_{f}+\alpha_{p} u_{p}\right)=-\nabla_{x} \cdot\left(\left(1-\alpha_{p}\right) u_{f}+\alpha_{p} u_{p}\right) .
$$

This constraint - the mean volume velocity is divergence free - can be seen as a definition of the pressure $P$.

- Secondly, the total momentum satisfies

$$
\begin{aligned}
\partial_{t}\left(\alpha_{f} \varrho_{f} u_{f}+\alpha_{p} \varrho_{p} u_{p}\right)+\nabla_{x} & \cdot\left(\alpha_{f} \varrho_{f} u_{f} \otimes u_{f}+\varrho_{p} \mathbb{P}\right) \\
& -\nabla_{x} \cdot\left(\alpha_{f} \tau\right)+\nabla_{x}\left(P+\mathrm{P}_{\star} \pi\left(\alpha_{p}\right)\right)=\left(\alpha_{f} \varrho_{f}+\alpha_{p} \varrho_{p}\right) g .
\end{aligned}
$$

Remark 2.1 Note that in many situations, hydrodynamic systems for inviscid fluid are used: viscosity effects are significant on the scale of the particles (it enters in the definition of the drag force) but can be disregarded at the macroscopic scale of the fluid. 


\subsubsection{Energy Dissipation}

Let us now show that the system (7)-(10) satisfies a remarkable dissipation property. Let us introduce the gravity potential $\mathscr{G}$ such that

$$
g=-\nabla_{x} \mathscr{G} .
$$

It is also convenient to set

$$
c=\sqrt{\frac{\mathrm{P}_{\star}}{\varrho_{p}}}
$$

which has the homogeneity of a velocity. We introduce the function $\varphi$, from $\left(0, \alpha_{\star}\right)$ to $\mathbb{R}$, as the primitive of $\alpha \mapsto c^{2} \pi(\alpha) / \alpha^{2}$ such that $\varphi\left(\alpha_{0}\right)=0$ for a given $\alpha_{0} \in\left(0, \alpha_{\star}\right)$. Owing to the assumption (i) of (4), $\varphi$ is an increasing function of class $\mathcal{C}^{2}$ and, since $\pi(0)=0$, we have $\alpha \varphi(\alpha) \rightarrow 0$ when $\alpha \rightarrow 0$. We next define the function $\Phi$ from $\left[0, \alpha_{\star}\right)$ to $[0,+\infty)$ by $\Phi(0)=0$ and $\Phi(\alpha)=\alpha \varphi(\alpha), \forall \alpha \in\left(0, \alpha_{\star}\right)$. It satisfies

(i) $\Phi \in \mathcal{C}^{0}\left(\left[0, \alpha_{\star}\right)\right) \cap \mathcal{C}^{2}\left(\left(0, \alpha_{\star}\right)\right), \quad \Phi(0)=0 \quad$ and $\quad \Phi(\alpha) \geq 0, \forall \alpha \in\left(\alpha_{0}, \alpha_{\star}\right)$,

(ii) $\alpha \Phi^{\prime}(\alpha)=\Phi(\alpha)+c^{2} \pi(\alpha), \forall \alpha \in\left(0, \alpha_{\star}\right)$,

(iii) $\Phi^{\prime \prime}(\alpha)=\frac{c^{2}}{\alpha} \pi^{\prime}(\alpha), \forall \alpha \in\left(0, \alpha_{\star}\right)$.

From (i) and (ii), we deduce that $\Phi$ is bounded from below and that it is an increasing function at least for $\alpha \in\left(\alpha_{0}, \alpha_{\star}\right)$. From (iii), we deduce that $\Phi$ is a convex function.

Remark 2.2 For the specific law (5), when $\beta=2$, we have

$$
\Phi(\alpha)=-c^{2} \alpha \ln \left(\alpha_{\star}-\alpha\right),
$$

and when $\beta=3$, we have

$$
\Phi(\alpha)=-c^{2} \alpha^{2}-c^{2} \alpha \alpha_{\star} \ln \left(\alpha_{\star}-\alpha\right) .
$$

It is remarkable that the PDE system dissipates energy, as established in the following statement, the proof of which is postponed to the Appendix A.

Proposition 2.3 Let $\left(f, \alpha_{f}, u_{f}, P\right)$ be a smooth enough solution of (7)-(10), with $0 \leq$ $\alpha_{p}(t, x)<\alpha_{\star}$. We assume that $f$ vanishes for large $|\xi|$. We define the following macroscopic energies

$$
\mathscr{E}_{f}=\frac{1}{2} \alpha_{f} \varrho_{f}\left|u_{f}\right|^{2}+\alpha_{f} \varrho_{f} \mathscr{G}, \quad \mathscr{E}_{p}=\frac{4}{3} \pi \bar{a}^{3} \varrho_{p} \int \frac{|\xi|^{2}}{2} f \mathrm{~d} \xi+\alpha_{p} \varrho_{p} \mathscr{G}+\varrho_{p} \Phi\left(\alpha_{p}\right) .
$$

and the following energy fluxes

$$
\begin{gathered}
\mathscr{H}_{f}=\mathscr{E}_{f} u_{f}-\alpha_{f} \tau . u_{f}+\alpha_{f} P u_{f}, \\
\mathscr{H}_{p}=\frac{4}{3} \pi \bar{a}^{3} \varrho_{p} \int \xi \frac{|\xi|^{2}}{2} f \mathrm{~d} \xi+\alpha_{p} \varrho_{p} \mathscr{G} u_{p}+\varrho_{p} \Phi\left(\alpha_{p}\right) u_{p}+\left(\alpha_{p} P+\pi\left(\alpha_{p}\right) P_{\star}\right) u_{p} .
\end{gathered}
$$

Then, the following energy equation holds

$$
\begin{aligned}
\partial_{t}\left(\mathscr{E}_{f}+\mathscr{E}_{p}\right)+\nabla_{x} \cdot\left(\mathscr{H}_{f}+\mathscr{H}_{p}\right) \\
\quad+\frac{4}{3} \pi \bar{a}^{3} \varrho_{p} D_{p} \int\left(\xi-u_{f}\right)^{2} f \mathrm{~d} \xi+\mu \alpha_{f}\left(\frac{1}{2}\left|\nabla_{x} u_{f}+\left(\nabla_{x} u_{f}\right)^{\top}\right|^{2}-\frac{2}{3}\left|\nabla_{x} \cdot u_{f}\right|^{2}\right)=0 .
\end{aligned}
$$

Note that the last term in the left hand side is always positive (see Appendix A). 


\subsection{A Purely Hydrodynamic Model: Mono-Kinetic Par- ticles}

In $[1,2,35,34,41]$ the kinetic equation is used as a numerical device to update the influence of the particles through Particle-In-Cell approaches: only "macro-particles" are considered, intended to be representative of a set of physical particles, the trajectories of which obey the ODEs system

$$
\frac{\mathrm{d}}{\mathrm{d} t} X=V, \quad \frac{\mathrm{d}}{\mathrm{d} t} V=A(t, X, V, a) .
$$

In fact, for most of the applications, the kinetic model (9) is certainly over-complicated. One is rather interested in a simplified model of hydrodynamic type. When collisions or Brownian motion are taken into account, these phenomena introduce relaxation mechanisms which can allow us to derive such relevant hydrodynamic models $[10,23$, 24]. In what follows, we adopt a somehow more restrictive viewpoint by assuming that particles are mono-kinetic

$$
\frac{4}{3} \pi \bar{a}^{3} f(t, x, \xi)=\alpha_{p}(t, x) \delta\left(\xi=u_{p}(t, x)\right) .
$$

In particular the kinetic pressure tensor becomes $\mathbb{P}=\alpha_{p} u_{p} \otimes u_{p}$ and we obtain a closed system of PDEs where the unknowns $\left(\alpha_{p}, \alpha_{f}=1-\alpha_{p}, u_{f}, u_{p}, P\right)$ describe the mixture flows. Indeed, equations (7)-(10) become

$$
\begin{aligned}
& \partial_{t} \alpha_{p}+\nabla_{x} \cdot\left(\alpha_{p} u_{p}\right)=0, \\
& \partial_{t}\left(\alpha_{p} u_{p}\right)+\nabla_{x} \cdot\left(\alpha_{p} u_{p} \otimes u_{p}+c^{2} \pi\left(\alpha_{p}\right) \mathrm{Id}\right)+\frac{\alpha_{p}}{\varrho_{p}} \nabla_{x} P=\alpha_{p} D_{p}\left(u_{f}-u_{p}\right)+\alpha_{p} g, \\
& \partial_{t} \alpha_{f}+\nabla_{x} \cdot\left(\alpha_{f} u_{f}\right)=0, \\
& \partial_{t}\left(\alpha_{f} u_{f}\right)+\nabla_{x} \cdot\left(\alpha_{f} u_{f} \otimes u_{f}\right)-\frac{1}{\varrho_{f}} \nabla_{x} \cdot\left(\alpha_{f} \tau\right) \\
& \quad+\frac{\alpha_{f}}{\varrho_{f}} \nabla_{x} P=\frac{\varrho_{p}}{\varrho_{f}} D_{p} \alpha_{p}\left(u_{p}-u_{f}\right)+\alpha_{f} g, \\
& \alpha_{f}+\alpha_{p}=1, \quad 0 \leq \alpha_{f}, \alpha_{p} \leq 1,
\end{aligned}
$$

where we recall that the viscous stress tensor $\tau$ is defined by (1).

We refer the reader to $[21,32]$ for a thorough discussion of more intricate closure relations. By the way, relevant closures of higher order can be constructed based on superposition of mono-kinetic ansatz [31]. The hydrodynamic model (15)-(19) satisfies the following dissipation property, which is the analog to Proposition 2.3.

Proposition 2.4 Let $\left(\alpha_{p}, \alpha_{f}=1-\alpha_{p}, u_{f}, u_{p}, P\right)$ be a smooth solution of (15)-(19), with $0 \leq \alpha_{p}(t, x)<\alpha_{\star}$. We define the following energies

$$
\mathscr{E}_{f}=\frac{1}{2} \alpha_{f} \varrho_{f}\left|u_{f}\right|^{2}+\alpha_{f} \varrho_{f} \mathscr{G}, \quad \mathscr{E}_{p}=\frac{1}{2} \alpha_{p} \varrho_{p}\left|u_{p}\right|^{2}+\alpha_{p} \varrho_{p} \mathscr{G}+\varrho_{p} \Phi\left(\alpha_{p}\right) .
$$


Then, the following energy equation holds

$$
\begin{aligned}
\partial_{t}\left(\mathscr{E}_{f}+\mathscr{E}_{p}\right) & +\nabla_{x} \cdot\left(\mathscr{E}_{f} u_{f}-\alpha_{f} \tau . u_{f}+\alpha_{f} P u_{f}+\mathscr{E}_{p} u_{p}+\left(\alpha_{p} P+\pi\left(\alpha_{p}\right) P_{\star}\right) u_{p}\right) \\
& +\alpha_{p} \varrho_{p} D_{p}\left(u_{p}-u_{f}\right)^{2}+\mu \alpha_{f}\left(\frac{1}{2}\left|\nabla_{x} u_{f}+\left(\nabla_{x} u_{f}\right)^{\top}\right|^{2}-\frac{2}{3}\left|\nabla_{x} \cdot u_{f}\right|^{2}\right)=0 .
\end{aligned}
$$

The arguments to justify this statement are detailed in the Appendix. Let us discuss more precisely the role of the close-packing term where $\pi\left(\alpha_{p}\right)$ can be interpreted as a pressure functional. To understand the limiting role of this pressure and the conditions in (4), we consider the following single phase system

$$
\begin{aligned}
& \partial_{t} \alpha+\nabla_{x} \cdot(\alpha u)=0, \\
& \partial_{t}(\alpha u)+\nabla_{x} \cdot\left(\alpha u \otimes u+c^{2} \pi(\alpha)\right)=0 .
\end{aligned}
$$

It looks like gas dynamics systems, with a pressure law that blows up when the density tends to $\alpha_{\star}$. Gases obeying such a pressure law are refereed to as Bethe-Weyl gases; we refer the reader to [4] for details on the analysis of such systems. System (20)-(21) is supplemented by initial conditions:

$$
\left.(\alpha, \alpha u)\right|_{t=0}=\left(\alpha^{0}, \alpha^{0} u^{0}\right),
$$

with $0 \leq \alpha^{0}(x)<\alpha_{\star}$. The close-packing pressure is intended to maintain the volume fraction $\alpha$ below the critical threshold $\alpha_{\star}$. Let us give some hints in this direction, for the system (20)-(21). In what follows, we still denote $q=\alpha u$. Let us define the flux function

$$
(\alpha, q) \mapsto F(\alpha, q)=\left(\begin{array}{c}
\alpha u^{\top} \\
\alpha u \otimes u+c^{2} \pi(\alpha) \mathrm{Id}
\end{array}\right)=\left(\begin{array}{c}
q^{\top} \\
q \otimes q / \alpha+c^{2} \pi(\alpha) \mathrm{Id}
\end{array}\right) .
$$

where $u^{\top}$ and $q^{\top}$ stand for the transposes of the column vectors $u$ and $q$.

Let us restrict our discussion to the one dimensional case for a moment, that is we assume that the space dimension variable $x$ belongs to $\mathbb{R}$ and that the velocity $u$ has only one component (again denoted by $u$ ). The flux $F(\alpha, q) \in \mathbb{R}^{2}$ is vector valued and we have

$$
\nabla_{\alpha, q} F(\alpha, \alpha u)=\left(\begin{array}{cc}
0 & 1 \\
-u^{2}+c^{2} \pi^{\prime}(\alpha) & 2 u
\end{array}\right)
$$

The eigenvalues of this matrix are

$$
\lambda_{-}(\alpha, u)=u-c \sqrt{\pi^{\prime}(\alpha)} \quad \text { and } \quad \lambda_{+}(\alpha, u)=u+c \sqrt{\pi^{\prime}(\alpha)} .
$$

The associated Riemann invariant of the system (20)-(21) are then

$$
\omega_{+}(\alpha, u)=u+G(\alpha) \quad \text { and } \quad \omega_{-}(\alpha, u)=u-G(\alpha)
$$

where

$$
G^{\prime}(\alpha)=\frac{c}{\alpha} \sqrt{\pi^{\prime}(\alpha)}
$$


As far as the solutions of (20)-(21) are smooth, they satisfy $\partial_{t} \omega_{ \pm}+\lambda_{ \pm} \partial_{x} \omega_{ \pm}=0$. Owing to (4), $\alpha \mapsto \alpha G(\alpha)$ is convex. Hence, for any $\kappa \in \mathbb{R}$, the sets $\{(\alpha, u), u+G(\alpha) \leqslant \kappa\}$ and $\{(\alpha, \alpha u), u-G(\alpha) \geqslant \kappa\}$ are convex and they are left invariant by the dynamics, see for instance [40, Th. 8.3.8]. This observation provides uniform estimates on the solutions of (20)-(21). Accordingly, since (4) implies that $G$ blows up as $\alpha \rightarrow \alpha_{\star}$, we can deduce $L^{\infty}$ estimates on the volume fraction $\alpha$ (far from $\alpha_{\star}$ ) and the velocity $u$, by means of the initial data. We refer for instance to [15] for the analysis of such invariant sets through viscous approximations.

In the three dimensional case, the situation is obviously more complex. We can obtain estimates on the characteristic speeds as follows. We write (20)-(21) in nonconservative form: with $U=(\alpha, q) \in \mathbb{R}^{N+1}$, we have

$$
\partial_{t} U_{m}+\sum_{j=1}^{N} \sum_{\ell=1}^{N+1} \partial_{U_{\ell}} F(U)_{m, j} \partial_{x_{j}} U_{\ell}=0, \quad \forall m=1, \ldots, N+1 .
$$

For $k \in \mathbb{R}^{N} \backslash\{0\}$, we consider the $(N+1) \times(N+1)$ matrix with components $A(U, k)_{m, \ell}=$ $\sum_{j=1}^{N} \partial_{U_{\ell}} F(U)_{m, j} k_{j}$. It can be checked that the eigenvalues of $A(U, k)$ are

$\lambda_{-}(U, k)=|k|\left(u \cdot \frac{k}{|k|}-c \sqrt{\pi^{\prime}(\alpha)}\right), \quad \lambda_{0}(U, k)=u \cdot k, \quad \lambda_{+}(U, k)=|k|\left(u \cdot \frac{k}{|k|}+c \sqrt{\pi^{\prime}(\alpha)}\right)$

the eigenspaces being of dimension $1, N-1,1$, respectively. In particular, we observe that the eigenvalues remain in the interval $\left[-|k|\left(|u|-\sqrt{\pi^{\prime}(\alpha)}\right),|k|\left(|u|+\sqrt{\pi^{\prime}(\alpha)}\right)\right]$.

\section{A numerical scheme for the coupled problem}

We are interested in the numerical approximation of the system (15)-(19), which is seen as a rough purely hydrodynamic model for describing the mixture. Similar macroscopic models for mixture flows are devised in [29, 38] and [39, Chap. $8 \& 10]$. We do not discuss further the modeling issues; instead we focus now on the numerical treatment of the PDEs system (15)-(19). The system is supplemented here with no-slip boundary conditions, that is

$$
u_{p}=0 \quad \text { and } \quad u_{f}=0 \quad \text { on } \partial \Omega .
$$

We describe the principles of the scheme for the coupled problem by focusing first on the time discretization. We make the treatment of the drag force implicit because it is usually a stiff term in the model. Hence, our strategy follows the method introduced in $[26,27]$, the new difficulties being related to the close-packing term and the fact that we account for the volume occupied by the particles at the scale of the fluid, which leads to the constraint $\alpha_{p}+\alpha_{f}=1$ for defining the pressure. The latter is treated in the spirit of projection methods for incompressible viscous flows, as introduced by A. Chorin [12, 13, 14] and R. Temam [42]. We refer to [28] for an documented overview on projection methods. However we shall see that space discretization leads to difficulties in order to preserve at the discrete level the mass conservation. To this end, the scheme we propose relies on the following ingredients: 
- We work with staggered grids, where volume fractions, pressure and velocities are evaluated on different grids,

- We adopt the Finite Volume framework and for the definition of the numerical fluxes, we adapt to our purposes the so-called kinetic schemes, as in [6].

In turn, we are able to provide some arguments for the stability analysis of the scheme, with respect to the positivity of the volume fractions, the close-packing limit, and the decay of the total entropy.

\subsection{Time discretization : incremental projection method}

We start with the time discretization. At the time $t^{k}, k \geq 0$, of the time marching, we have at hand $\alpha_{p}^{k}, q_{p}^{k}=\alpha_{p}^{k} u_{p}^{k}, \alpha_{f}^{k}, u_{f}^{k}, P^{k}$ such that

$$
\begin{aligned}
& \alpha_{p}^{k}+\alpha_{f}^{k}=1, \\
& \nabla_{x} \cdot\left(\alpha_{p}^{k} u_{p}^{k}+\alpha_{f}^{k} u_{f}^{k}\right)=0 .
\end{aligned}
$$

To compute these quantities at time $t^{k+1}=t^{k}+\delta t$, we proceed as follows:

- We start by updating the volume fractions with the transport equation

$$
\begin{gathered}
\frac{\alpha_{p}^{k+1}-\alpha_{p}^{k}}{\delta t}=-\nabla_{x} \cdot\left(\alpha_{p}^{k} u_{p}^{k}\right), \\
\frac{\alpha_{f}^{k+1}-\alpha_{f}^{k}}{\delta t}=-\nabla_{x} \cdot\left(\alpha_{f}^{k} u_{f}^{k}\right) .
\end{gathered}
$$

Note that, since $\alpha_{p}^{k}, u_{p}^{k}, \alpha_{f}^{k}$ and $u_{f}^{k}$ satisfy the conditions (23) and (24), we can arbitrarily replace one of the previous equations by $\alpha_{f}^{k+1}+\alpha_{p}^{k+1}=1$.

- Then, we update the momentum equations: with the drag force considered implicitly, we predict the velocities by solving the following system

$$
\begin{aligned}
\frac{\widetilde{q_{p}}-q_{p}^{k}}{\delta t}+D_{p}\left(\widetilde{q_{p}}-\right. & \left.\alpha_{p}^{k+1} \widetilde{u_{f}}\right) \\
& =-\nabla_{x} \cdot\left(q_{p}^{k} \otimes q^{k} / \alpha_{p}^{k}\right)-c^{2} \nabla_{x} \pi\left(\alpha_{p}^{k}\right)-\frac{\alpha_{p}^{k}}{\varrho_{p}} \nabla_{x} \widetilde{P}+\alpha_{p}^{k+1} g, \\
\frac{\alpha_{f}^{k+1} \widetilde{u_{f}}-\alpha_{f}^{k} u_{f}^{k}}{\delta t}+ & \frac{\varrho_{p}}{\varrho_{f}} D_{p}\left(\alpha_{p}^{k+1} \widetilde{u_{f}}-\widetilde{q_{p}}\right)-\frac{1}{\varrho_{f}} \nabla_{x} \cdot\left(\alpha_{f}^{k+1} \widetilde{\tau}\right) \\
& =-\nabla_{x} \cdot\left(\alpha_{f}^{k} u_{f}^{k} \otimes u_{f}^{k}\right)-\frac{\alpha_{f}^{k}}{\varrho_{f}} \nabla_{x} \widetilde{P}+\alpha_{f}^{k+1} g .
\end{aligned}
$$

where $\widetilde{\tau}=\mu\left(\nabla_{x} \widetilde{u_{f}}+\left(\nabla_{x} \widetilde{u_{f}}\right)^{\top}-\frac{2}{3}\left(\nabla_{x} \cdot \widetilde{u_{f}}\right) \mathrm{Id}\right)$.

In these equations the pressure $\widetilde{P}$ is given by one of the following options:

$$
\text { either } \widetilde{P}=0 \text { or } \widetilde{P}=P^{k} \text {. }
$$

This allows us to present in a single formulation a non incremental $(\widetilde{P}=0)$ or an incremental $\left(\widetilde{P}=P^{k}\right)$ version of the algorithm. 
- We finally update the pressure and correct the velocities. To this end, we remind the reader that the mean volume velocity $W=\alpha_{p} u_{p}+\left(1-\alpha_{p}\right) u_{f}$ is divergence free. Hence, we seek an auxilliary potential $\Psi$ such that

$$
\alpha_{f}^{k+1} u_{f}^{k+1}=\alpha_{f}^{k+1} \widetilde{u_{f}}-\alpha_{f}^{k+1} \frac{\delta t}{\varrho_{f}} \nabla_{x} \Psi, \quad q_{p}^{k+1}=\widetilde{q_{p}}-\alpha_{p}^{k+1} \frac{\delta t}{\varrho_{p}} \nabla_{x} \Psi
$$

satisfies $\nabla_{x} \cdot\left(q^{k+1}+\alpha_{f}^{k+1} u_{f}^{k+1}\right)=0$. It leads to the following diffusion like equation for the potential $\Psi$

$$
-\nabla_{x} \cdot\left(\left(\frac{\alpha_{p}^{k+1}}{\varrho_{p}}+\frac{\alpha_{f}^{k+1}}{\varrho_{f}}\right) \nabla_{x} \Psi\right)=-\frac{1}{\delta t} \nabla_{x} \cdot\left(\widetilde{q_{p}}+\alpha_{f}^{k+1} \widetilde{u_{f}}\right) .
$$

Having obtained the potential we can update the velocity $u_{f}^{k+1}$ and $u_{p}{ }^{k+1}$ and the pressure by $P^{k+1}=\widetilde{P}+\Psi$. With $\widetilde{P}=0$ we directly obtain the updated pressure (non-incremental method), while with $\widetilde{P}=P^{k}$ we compute the pressure increment (incremental method), and the pressure is accounted for in the prediction step.

We refer the reader to [28, Section 5] for further details on such methods for the Incompressible Navier-Stokes system, in particular for improvements of the time discretization (e. g. based on the BDF scheme).

\subsection{Space discretization in $1 \mathrm{D}$}

To avoid any odd/even decoupling in the stencil of the discrete version of the problem (28), we find convenient to work with staggered grids. It is worth mentioning similar ideas used when dealing with Low-Mach regimes in fluid mechanics, see [30] and the references therein. We present the framework in the one dimension case (but the method can be adapted to higher dimensions, at least this is quite straightforward when dealing with Cartesian meshes). We consider a set of $J+1$ points which defines a subdivison of the computational domain $[0, L]: x_{1}=0<x_{2}<\ldots<x_{J}<x_{J+1}=L$. This is the primal mesh. We set

$$
\delta x_{j+1 / 2}=x_{j+1}-x_{j} .
$$

Next, let $x_{j+1 / 2}=\frac{x_{j}+x_{j+1}}{2}$. The $J$ points $x_{3 / 2}<\ldots<x_{J+1 / 2}$ define the dual mesh, where we set $\delta x_{1}=\delta x_{3 / 2} / 2, \delta x_{J}=\delta x_{J+1 / 2} / 2$ and

$$
\delta x_{j}=\frac{\delta x_{j-1 / 2}+\delta x_{j+1 / 2}}{2}
$$

for $j \in\{1, \ldots, J-1\}$. For the numerical unknowns:

- Volume fractions and pressure are evaluated on the dual mesh: $\alpha_{p, j+1 / 2}, \alpha_{f, j+1 / 2}$, $p_{j+1 / 2}$, with $j \in\{1, \ldots, J\}$,

- Velocities are evaluated on the primal mesh $u_{f, j}, u_{p, j}$ with $j \in\{1, \ldots, J+1\}$.

We recall that we wish to impose no-slip boundary conditions (22) so that we impose

$$
u_{p, 1}^{k}=u_{p, J+1}^{k}=0, \quad \text { and } \quad u_{f, 1}^{k}=u_{f, J+1}^{k}=0, \quad \text { for all } k .
$$




\subsubsection{Discretization of the prediction step}

The two volume fractions and momentums are updated with Finite Volume approximations. The gradient of the pressure $\nabla_{x} \widetilde{P}$ is approximated by a centered scheme

$$
\left(\nabla_{x} \widetilde{P}\right)_{j}=\frac{\widetilde{P}_{j+1 / 2}-\widetilde{P}_{j-1 / 2}}{\delta x_{j}}, \quad j=2, \ldots, J,
$$

and the space discretization of the zeroth order terms (friction and gravity) is trivial. The diffusive flux $\alpha_{f} \tau$ is implicitly discretized as follows

$$
\frac{4}{3} \mu \alpha_{f, j+1 / 2}^{k} \frac{u_{f, j+1}^{k+1}-u_{f, j}^{k+1}}{\delta x_{j+1 / 2}}, \quad j=2, \ldots, J .
$$

We are now going to derive a relevant definition of the convection fluxes by means of the kinetic approach.

We refer the reader to $[9,19,36,37]$ for further details on the so-called kinetic schemes. An application for particulate flows is discussed in [25]. Roughly speaking, in this framework numerical fluxes are defined as integrals over an auxilliary variable $\xi$ of a certain "equilibrium function" $\mathscr{M}$, according to UpWinding principles. The analysis of the scheme then relies on the properties of the equilibrium function. In what follows, we adapt this method for designing numerical fluxes for updating $\alpha_{p}$ and $u_{p}$ in (15)(19). In fact, to start with, let us just consider (20)-(21), postponing for a while the difficulties related to the treatment of the coupling. We use the kinetic schemes on staggered grids designed in [6] for general barotropic Euler equations. We set

$$
\begin{aligned}
M_{0}(\alpha, u, \xi) & =\frac{\alpha}{2 c \sqrt{\pi^{\prime}(\alpha)}} \mathbf{1}_{|\xi-u| \leq c \sqrt{\pi^{\prime}(\alpha)}}, \\
M_{1}(\alpha, u, \xi) & =u M_{0}(\alpha, u, \xi)+\tilde{M}(\alpha, u, \xi),
\end{aligned}
$$

where $\mathbf{1}_{A}$ stands for the characteristic function of the set $A$ and

$$
\begin{aligned}
& \tilde{M}(\alpha, u, \xi)=\xi L(\alpha, u) \mathbf{1}_{|\xi| \leq|u|+c \sqrt{\pi^{\prime}(\alpha)}} \\
& L(\alpha, u)=\frac{3}{2} \frac{c^{2} \pi(\alpha)}{\left(|u|+c \sqrt{\pi^{\prime}(\alpha)}\right)^{3}} .
\end{aligned}
$$

Flux-consistency is guaranteed by the following relations (see [6, Prop. 3.1])

$$
\begin{aligned}
\int(1, \xi) M_{0}\left(\alpha_{p}, u_{p}, \xi\right) \mathrm{d} \xi & =\left(\alpha_{p}, \alpha_{p} u_{p}\right) \\
\int(1, \xi) M_{1}\left(\alpha_{p}, u_{p}, \xi\right) \mathrm{d} \xi & =\left(\alpha_{p} u_{p}, \alpha_{p} u_{p}^{2}+c^{2} \pi\left(\alpha_{p}\right)\right) .
\end{aligned}
$$

For updating the density $\alpha_{p}$, the scheme reads

$$
\frac{\delta x_{j+1 / 2}}{\delta t}\left(\alpha_{p, j+1 / 2}^{k+1}-\alpha_{p, j+1 / 2}^{k}\right)+\mathscr{F}_{j+1}^{k}-\mathscr{F}_{j}^{k}=0, \quad j=1, \ldots, J .
$$


The numerical fluxes are given by

$$
\mathscr{F}_{1}^{k}=0, \quad \mathscr{F}_{J+1}^{k}=0, \quad \mathscr{F}_{j}^{k}=\mathscr{F}^{+}\left(\alpha_{p, j-1 / 2}^{k}, u_{p, j}^{k}\right)+\mathscr{F}^{-}\left(\alpha_{p, j+1 / 2}^{k}, u_{p, j}^{k}\right), \quad j=2, \ldots, J,
$$

where the mappings $\mathscr{F}^{ \pm}$are defined by

$$
\mathscr{F}^{ \pm}\left(\alpha_{p}, u_{p}\right)=\int_{\xi \gtrless 0} \xi M_{0}\left(\alpha_{p}, u_{p}, \xi\right) \mathrm{d} \xi .
$$

Quite naturally, we use the value of the velocity at the interface $x=x_{j}$ and we upwind the density. We point out that in practice we do not have to appeal to a numerical procedure in order to evaluate the integrals. Indeed, the fluxes are given by the following simple formula

$$
\mathscr{F}^{+}\left(\alpha_{p}, u_{p}\right)= \begin{cases}0 & \text { if } u_{p}+c\left(\alpha_{p}\right) \leqslant 0 \\ \frac{\alpha_{p}}{4 c\left(\alpha_{p}\right)}\left(u_{p}+c\left(\alpha_{p}\right)\right)^{2} & \text { if } u_{p}-c\left(\alpha_{p}\right)<0<u_{p}+c\left(\alpha_{p}\right), \\ \alpha_{p} u_{p} & \text { if } 0<u_{p}-c\left(\alpha_{p}\right),\end{cases}
$$

and

$$
\mathscr{F}^{-}\left(\alpha_{p}, u_{p}\right)= \begin{cases}\alpha_{p} u_{p} & \text { if } u_{p}+c\left(\alpha_{p}\right) \leqslant 0 \\ -\frac{\alpha_{p}}{4 c\left(\alpha_{p}\right)}\left(u_{p}-c\left(\alpha_{p}\right)\right)^{2} & \text { if } u_{p}-c\left(\alpha_{p}\right)<0<u_{p}+c\left(\alpha_{p}\right) \\ 0 & \text { if } 0<u_{p}-c\left(\alpha_{p}\right)\end{cases}
$$

The expression of the mass fluxes being given, we can define the discrete counterpart $\mathbb{D}^{(1)}$ of $\partial_{x}\left(\alpha_{p} u_{p}\right)$ (which is located at cell center) as follows

$$
\left[\mathbb{D}^{(1)}\left(\alpha_{p}, u_{p}\right)\right]_{j+1 / 2}=\frac{\mathscr{F}_{j+1}-\mathscr{F}_{j}}{\delta x_{j+1 / 2}}, \quad j=1, \ldots, J .
$$

In order to define a Finite Volume approximation of the momentum equation on the cells $\left(x_{j-1 / 2}, x_{j+1 / 2}\right)$ of the dual mesh, we proceed as follows. We introduce

$$
\alpha_{p, j}^{k}=\frac{\delta x_{j+1 / 2} \alpha_{p, j+1 / 2}^{k}+\delta x_{j-1 / 2} \alpha_{p, j-1 / 2}^{k}}{2 \delta x_{j}}, \quad j=2, \ldots, J .
$$

This is the mean value of the volume fraction on the cell $\left(x_{j-1 / 2}, x_{j+1 / 2}\right)$. We update the velocity with

$$
\frac{\delta x_{j}}{\delta t}\left(\alpha_{p, j}^{k+1} u_{p, j}^{k+1}-\alpha_{p, j}^{k} u_{p, j}^{k}\right)+\widetilde{\mathscr{F}}_{j+1 / 2}^{k}-\widetilde{\mathscr{F}}_{j-1 / 2}^{k}=\text { Source terms, } \quad j=2, \ldots, J .
$$

The fluxes are defined by

$$
\begin{aligned}
\widetilde{\mathscr{F}}_{j+1 / 2}^{k}= & \frac{u_{p, j}^{k}}{2}\left(\mathscr{F}^{+}\left(\alpha_{p, j-1 / 2}^{k}, u_{p, j}^{k}\right)+\mathscr{F}^{+}\left(\alpha_{p, j+1 / 2}^{k}, u_{p, j+1}^{k}\right)\right) \\
& +\frac{u_{p, j+1}^{k}}{2}\left(\mathscr{F}^{-}\left(\alpha_{p, j+1 / 2}^{k}, u_{p, j}^{k}\right)+\mathscr{F}^{-}\left(\alpha_{p, j+3 / 2}^{k}, u_{p, j+1}^{k}\right)\right)+c^{2} \pi\left(\alpha_{p, j+1 / 2}^{k}\right) .
\end{aligned}
$$


This formula applies for $j \in\{2, \ldots, J-1\}$. Namely, the discretization of the closepacking term is centered at $x_{j}$, while we upwind the product $\alpha_{p} u_{p} \times u_{p}$ by using the (known) mass fluxes. Precisely, we consider the average of the positive mass fluxes $\mathscr{F}^{+}$ and the average of the negative mass fluxes $\mathscr{F}^{-}$known at $x_{j}$ and $x_{j+1}$. For $j=1$, (resp. $j=J$ ) we remind the reader that $u_{p, 1}^{k}=0$ (resp. $u_{p, J+1}^{k}=0$ ) so that the contribution associated to the positive (resp. negative) $\xi$ 's vanishes. Hence only the mass flux from right to left at the interface $x_{3 / 2}$ (resp. the mass flux from left to right at the interface $x_{J-1 / 2}$ ) has to be considered. Since there is no mass flux at $x=0$ (resp. $x=L$ ), we arrive at

$$
\begin{aligned}
& \widetilde{\mathscr{F}}_{3 / 2}^{k}=\frac{u_{p, 2}^{k}}{2} \mathscr{F}^{-}\left(\alpha_{p, 5 / 2}^{k}, u_{p, 2}^{k}\right)+c^{2} \pi\left(\alpha_{p, 5 / 2}^{k}\right), \\
& \widetilde{\mathscr{F}}_{J+1 / 2}^{k}=\frac{u_{p, J}^{k}}{2} \mathscr{F}^{+}\left(\alpha_{p, J-1 / 2}^{k}, u_{p, J}^{k}\right)+c^{2} \pi\left(\alpha_{p, J-1 / 2}^{k}\right) .
\end{aligned}
$$

As a matter of fact, we check the consistency of the scheme.

Proposition 3.1 The flux decomposition is consistent since $\mathscr{F}^{+}\left(\alpha_{p}, u_{p}\right)+\mathscr{F}^{-}\left(\alpha_{p}, u_{p}\right)=$ $\alpha_{p} u_{p}$ holds.

Remark 3.2 Note that $M_{0}$ and $M_{1}$ are not usual formulae for the equilibrium state. Beyond the support property, the identification of a relevant equilibrium state can be based on the entropy property: it relies to minimize an entropy functional, associated to the entropy of the system of conservation laws, with moments constraints. For isentropic gas dynamics, finding the explicit solution of the minimization problem uses the homogeneity of the pressure law, see [9, Section 2.5], [8] and [37, Prop. 1.4.1 and Section 8]. It seems difficult to identify the equilibrium state by this method when dealing with a general pressure law. Nevertheless, the scheme detailed below can be shown to satisfy a Lax-Wendroff-like consistency statement [5].

Going back to the coupling, we introduce similarly suitable flux functions for the fluid density and momentum $\mathscr{G}_{j}^{k}$ and $\widetilde{\mathscr{G}}_{j+1 / 2}^{k}$. For the transport terms of fluid equations, we use an UpWinding strategy based only on the material velocity $u_{f}$, that is we use the following definitions

$$
\mathscr{G}_{j}^{k}=\mathscr{F}^{\mathrm{UpW},+}\left(\alpha_{f, j-1 / 2}^{k}, u_{f, j}^{k}\right)+\mathscr{F}^{\mathrm{UpW},-}\left(\alpha_{f, j+1 / 2}^{k}, u_{f, j}^{k}\right),
$$

and

$$
\begin{aligned}
\widetilde{\mathscr{G}}_{j+1 / 2}^{k}= & \frac{u_{f, j}^{k}}{2}\left(\mathscr{F} \mathrm{UpW},+\left(\alpha_{f, j-1 / 2}^{k}, u_{f, j}^{k}\right)+\mathscr{F}^{\mathrm{UpW},+}\left(\alpha_{f, j+1 / 2}^{k}, u_{f, j+1}^{k}\right)\right) \\
& +\frac{u_{f, j+1}^{k}}{2}\left(\mathscr{F} \mathrm{UpW},-\left(\alpha_{f, j+1 / 2}^{k}, u_{f, j}^{k}\right)+\mathscr{F} \mathrm{UpW},-\left(\alpha_{f, j+3 / 2}^{k}, u_{f, j+1}^{k}\right)\right),
\end{aligned}
$$

with

$$
\mathscr{F}^{\mathrm{UpW},+}\left(\alpha_{f}, u_{f}\right)= \begin{cases}0 & \text { if } u_{f} \leqslant 0 \\ \alpha_{f} u_{f} & \text { if } 0<u_{f}\end{cases}
$$


and

$$
\mathscr{F}^{\mathrm{UpW},-}\left(\alpha_{f}, u_{f}\right)= \begin{cases}\alpha_{f} u_{f} & \text { if } u_{f} \leqslant 0, \\ 0 & \text { if } 0<u_{f} .\end{cases}
$$

The discrete version of $\partial_{x}\left(\alpha_{f} u_{f}\right)$ is thus defined by a function

$$
\left[\mathbb{D}^{(2)}\left(\alpha_{f}, u_{f}\right)\right]_{j+1 / 2}=\frac{\mathscr{G}_{j+1}-\mathscr{G}_{j}}{\delta x_{j+1 / 2}}, \quad j=1, \ldots, J .
$$

\subsubsection{Discretization of the correction step}

At first sight, it might look reasonable to directly discretize the diffusion equation (28). For instance, in the one dimensional framework, on an uniform mesh, it leads to a formula which looks like

$$
\begin{aligned}
\frac{\delta t}{\delta x^{2}}\left(\frac{\nu_{j+1}^{k}+\nu_{j}^{k}}{2} \Psi_{j+1}-2 \nu_{j}^{k} \Psi_{j}+\frac{\nu_{j}^{k}+\nu_{j-1}^{k}}{2} \Psi_{j-1}\right) \\
=\text { Discrete version of } \partial_{x}\left(\alpha_{f}^{k+1} \widetilde{u_{f}}+\widetilde{q_{p}}\right)
\end{aligned}
$$

with $\nu_{j}^{k}=\alpha_{p, j}^{k} / \varrho_{p}+\alpha_{f, j}^{k} / \varrho_{f}$. In fact, further simplification can be used in 1D because at the continuous level the constraint simply means

$$
\alpha_{f} u_{f}+\alpha_{p} u_{p}=0
$$

since the boundary conditions impose vanishing velocities. This formula could also be directly used to find the gradient of the potential

$$
\widetilde{W}=\delta t\left(\frac{\alpha_{f}^{k+1}}{\varrho_{f}}+\frac{\alpha_{p}^{k+1}}{\varrho_{p}}\right) \partial_{x} \Psi,
$$

leading to $\alpha_{f}^{k+1} u_{f}^{k+1}+\alpha_{p}^{k+1} u_{p}^{k+1}=0$. Finite difference simulations based either on (34) (including results with two or three space dimensions) or (35) are presented in $[16,17]$ for models describing biofilms formations, which share many similarities with the system we are dealing with. We also refer the reader to [7] for two-dimensional simulations in the Finite Volume framework, still based on a direct discretization of the elliptic equation (34).

However, we have to bear in mind that the correction step in the time marching algorithm aims at ensuring the constraint (24) at time $t^{k+1}$. This equality is mandatory, at each time step, to ensure that, solving the two mass balance equations (25) and (26) or solving only one of these equations while using the constraint $\alpha_{f}+\alpha_{p}=1$ to update the other volume fraction, are strictly equivalent. At the discrete level in space, it means that we can independently choose suitable schemes to update the two volume fractions $\alpha_{f}$ and $\alpha_{p}$ while preserving the constraint $\alpha_{f}+\alpha_{p}=1$ (see Remark 3.3). To satisfy this property requires a careful strategy of space-discretization. Indeed, the updated volume fractions are defined by a numerical scheme which has the following general expression

$$
\alpha_{p, j+1 / 2}^{k+1}=\alpha_{p, j+1 / 2}^{k}-\frac{\delta t}{\delta x_{j+1 / 2}}\left(\mathscr{F}_{j+1}^{k}-\mathscr{F}_{j}^{k}\right), \quad \alpha_{f, j+1 / 2}^{k+1}=\alpha_{f, j+1 / 2}^{k}-\frac{\delta t}{\delta x_{j+1 / 2}}\left(\mathscr{G}_{j+1}^{k}-\mathscr{G}_{j}^{k}\right),
$$


where the numerical fluxes $\mathscr{F}_{j}^{k}$ (resp. $\mathscr{G}_{j}^{k}$ ) are suitable functions of $\alpha_{p, j-1 / 2}^{k}, \alpha_{p, j+1 / 2}^{k}$, $u_{p, j}^{k}\left(\right.$ resp. $\left.\alpha_{f, j-1 / 2}^{k}, \alpha_{f, j+1 / 2}^{k}, u_{f, j}^{k}\right)$. Therefore, the discrete version of the constraint $\partial_{t}\left(\alpha_{f}+\alpha_{p}\right)=0$ becomes

$$
\mathscr{F}_{j+1}^{k}-\mathscr{F}_{j}^{k}+\mathscr{G}_{j+1}^{k}-\mathscr{G}_{j}^{k}=0 .
$$

It corresponds to the discretization of $\partial_{x}\left(q_{p}+\alpha_{f} u_{f}\right)=0$, but the expression intimately depends on the definition of the numerical fluxes $\mathscr{F}_{j}^{k}, \mathscr{G}_{j}^{k}$. In general, it cannot be expressed as a simple flux function acting on the composite moment $q_{p}+\alpha_{f} u_{f}$. Consequently, if we define the correction $\Psi$ by solving (34) or by using (35), then, there is no reason to guarantee that $\mathscr{F}_{j+1}^{k+1}-\mathscr{F}_{j}^{k+1}+\mathscr{G}_{j+1}^{k+1}-\mathscr{G}_{j}^{k+1}=0$ holds. The definition of $\Psi$ should take into account the structure of the numerical fluxes $\mathscr{F}$ and $\mathscr{G}$.

Remark 3.3 The following example is quite illuminating. Let us choose

(i) an upwind scheme to update the volume fraction $\alpha_{p}$ :

$$
\alpha_{p, j+1 / 2}^{k+1}=\alpha_{p, j+1 / 2}^{k}-\frac{\delta t}{\delta x_{j+1 / 2}}\left(\mathscr{F}_{j+1}^{k}-\mathscr{F}_{j}^{k}\right), \text { with } \mathscr{F}_{j}^{k}=\left\{\begin{array}{cc}
\alpha_{p, j+1 / 2}^{k} & \text { if } u_{p, j}^{k} \leq 0, \\
\alpha_{p, j-1 / 2}^{k} & \text { if } u_{p, j}^{k} \geq 0
\end{array}\right.
$$

(ii) $\alpha_{p, j+1 / 2}^{k+1}=1-\alpha_{f, j+1 / 2}^{k+1}$ to update the volume fraction $\alpha_{f}$,

(iii) (35) to update the pressure

With these definitions, we are led to

$$
\alpha_{f, j+1 / 2}^{k+1}=\alpha_{f, j+1 / 2}^{k}-\frac{\delta t}{\delta x_{j+1 / 2}}\left(\mathscr{G}_{j+1}^{k}-\mathscr{G}_{j}^{k}\right), \text { with } \mathscr{G}_{j}^{k}= \begin{cases}\alpha_{f, j+1 / 2}^{k} & \text { if } u_{f, j}^{k} \geq 0, \\ \alpha_{f, j-1 / 2}^{k} & \text { if } u_{f, j}^{k} \leq 0\end{cases}
$$

since (iii) implies $u_{f}^{k}=-\frac{\alpha_{p}^{k}}{\alpha_{f}^{k}} u_{p}^{k}$. Thus, $\alpha_{f}$ is actually updated with a downwind scheme.

Using the notation (31) and (33), the discrete volume conservation constraint (36) thus reads

$$
\mathbb{D}^{(1)}\left(\alpha_{p}^{k}, u_{p}^{k}\right)+\mathbb{D}^{(2)}\left(\alpha_{f}^{k}, u_{f}^{k}\right)=0 .
$$

This relation must be satisfied at each time step. Using the expression (27) of the updated velocities $u_{p}^{k+1}$ and $u_{f}^{k+1}$, the discrete corrector $\Psi=\left(\Psi_{3 / 2}, \ldots, \Psi_{J+1 / 2}\right)$ is thus defined as the solution of

$$
\mathbb{D}^{(1)}\left(\alpha_{p}^{k+1}, \widetilde{u_{p}}-\frac{\delta t}{\varrho_{p}} \nabla^{D} \Psi\right)+\mathbb{D}^{(2)}\left(\alpha_{f}^{k+1}, \widetilde{u_{f}}-\frac{\delta t}{\varrho_{f}} \nabla^{D} \Psi\right)=0
$$

where $\nabla^{D}: \mathbb{R}^{J} \rightarrow \mathbb{R}^{J+1}$ is defined by $\nabla_{j}^{D} \Psi=\frac{\Psi_{j+1 / 2}-\Psi_{j-1 / 2}}{\delta x_{j}}$ for $j \in\{2, \ldots, J\}$ completed with the convention $\nabla_{1}^{D} \Psi=\nabla_{J+1}^{D} \Psi=0$ (these values are not involved in (37)). We point out that (37) is a non linear system. An abstract argument allows us to prove the existence of a solution.

Proposition 3.4 We suppose that $0<\underline{\alpha} \leq \alpha_{p, j+1 / 2}^{k+1} \leq \bar{\alpha}<\infty$ for any $j$. Then, the problem (37) admits a solution $\Psi \in \mathbb{R}^{J}$.

The proof is detailed in Appendix B. Numerically, we solve this system by using the standard Newton algorithm. Of course, it impacts the computational cost; in practice we observe that the solution is obtained in a few iterations. 


\subsubsection{Stability analysis}

The analysis of [6, Proposition 3.2] shows that the volume fractions remain positive, under a suitable CFL condition.

Lemma 3.5 Let $j \in\{1, . ., J\}$ and $k>0$. Assume that the time step $\delta t$ satisfies the CFL condition

$$
\begin{gathered}
\frac{\delta t}{\delta x_{j+1 / 2}}\left(\left[u_{f, j+1}^{k}\right]^{+}+\left[u_{f, j}^{k}\right]^{-}\right) \leqslant 1 . \\
\frac{\delta t}{\delta x_{j+1 / 2}}\left(\left[\lambda_{+}\left(\alpha_{p, j+1 / 2}^{k}, u_{p, j+1}^{k}\right)\right]^{+}+\left[\lambda_{-}\left(\alpha_{p, j+1 / 2}^{k}, u_{p, j}^{k}\right)\right]^{-}\right) \leqslant 1 .
\end{gathered}
$$

Then, the scheme preserves the positivity of $\alpha_{p}$ and $\alpha_{f}$, that is $\left(\alpha_{p, j+1 / 2}^{k}>0\right) \Rightarrow$ $\left(\alpha_{p, j+1 / 2}^{k+1}>0\right)$ and $\left(\alpha_{f, j+1 / 2}^{k}>0\right) \Rightarrow\left(\alpha_{f, j+1 / 2}^{k+1}>0\right)$.

We next turn to analyze the preservation of the close-packing constraint $\alpha_{p}<\alpha_{\star}$. Bearing in mind the following bounds (cf [6, Lemma 3.2 (i)(ii)]) on $\mathscr{F}^{ \pm}$

$$
\begin{aligned}
0 & \leqslant \mathscr{F}^{+}\left(\alpha_{p}, u_{p}\right) \leqslant \alpha_{p}\left[\lambda_{+}\left(\alpha_{p}, u_{p}\right)\right]^{+} \\
-\alpha_{p}\left[\lambda_{-}\left(\alpha_{p}, u_{p}\right)\right]^{-} & \leqslant \mathscr{F}^{-}\left(\alpha_{p}, u_{p}\right) \leqslant 0
\end{aligned}
$$

for all $u_{p} \in \mathbb{R}, \alpha_{p} \geqslant 0$, we estimate as follows

$$
\begin{aligned}
\alpha_{p, j+1 / 2}^{k+1} & \leqslant \alpha_{p, j+1 / 2}^{k}+\frac{\delta t}{\delta x_{j+1 / 2}}\left(\mathscr{F}^{+}\left(\alpha_{p, j-1 / 2}^{k}, u_{p_{j}}^{k}\right)-\mathscr{F}^{-}\left(\alpha_{p, j+3 / 2}^{k}, u_{p, j+1}^{k}\right)\right) \\
& \leqslant \alpha_{p, j+1 / 2}^{k}+\frac{\delta t}{\delta x_{j+1 / 2}}\left(\alpha_{p, j-1 / 2}^{k}\left[\lambda_{+}\left(\alpha_{p, j-1 / 2}^{k}, u_{p_{j}}^{k}\right)\right]^{+}+\alpha_{p, j+3 / 2}^{k}\left[\lambda_{-}\left(\alpha_{p, j+3 / 2}^{k}, u_{p, j+1}^{k}\right)\right]^{-}\right) .
\end{aligned}
$$

Now, let us assume $\alpha_{p, j+1 / 2}^{k}<\alpha_{\star}$, for all $j$. Let $\varepsilon_{j+1 / 2}^{k}=1-\alpha_{p, j+1 / 2}^{k} / \alpha_{\star}$. Then, we obtain

$\alpha_{p, j+1 / 2}^{k+1} \leqslant \alpha_{\star}-\alpha_{\star}\left[\varepsilon_{j+1 / 2}^{k}-\frac{\delta t}{\delta x_{j+1 / 2}}\left(\left[\lambda_{+}\left(\alpha_{p, j-1 / 2}^{k}, u_{p_{j}}^{k}\right)\right]^{+}+\left[\lambda_{-}\left(\alpha_{p, j+3 / 2}^{k}, u_{p, j+1}^{k}\right)\right]^{-}\right)\right]$.

Hence, we have $\alpha_{p, j+1 / 2}^{k+1}<\alpha_{\star}$ as soon as

$$
\frac{\delta t}{\delta x_{j+1 / 2}}\left(\left[\lambda_{+}\left(\alpha_{p, j-1 / 2}^{k}, u_{p_{j}}^{k}\right)\right]^{+}+\left[\lambda_{-}\left(\alpha_{p, j+3 / 2}^{k}, u_{p, j+1}^{k}\right)\right]^{-}\right)<\varepsilon_{j+1 / 2}^{k} .
$$

It allows to identify a constraint on the time step to preserve the close-packing threshold.

Proposition 3.6 Assume that $\alpha_{p, j+1 / 2}^{k}<\alpha_{\star}$, for all $j=1, . ., J$ and that the time step St satisfies the CFL condition

$$
\frac{\delta t}{\delta x_{j+1 / 2}}\left(\left[\lambda_{+}\left(\alpha_{p, j-1 / 2}^{k}, u_{p, j}^{k}\right)\right]^{+}+\left[\lambda_{-}\left(\alpha_{p, j+3 / 2}^{k}, u_{p, j+1}^{k}\right)\right]^{-}\right) \leqslant 1-\frac{\alpha_{p, j+1 / 2}^{k}}{\alpha_{\star}}, \quad \forall j=1, . ., J .
$$

Then, the scheme preserves the close-packing constraint $\alpha_{p}<\alpha_{\star}$, that is $\alpha_{p, j+1 / 2}^{k+1}<\alpha_{\star}$, for all $j=1, . ., J$. 
Unfortunately, the proof does not exclude the case where the numerical solution $\alpha_{p, j+1 / 2}^{k}$ gets closer to $\alpha_{\star}$ at each time step. When $\alpha_{p, j+1 / 2}^{k}$ goes to $\alpha_{\star}$, the condition (40) on the time step $\delta t$ becomes severe for two reasons : firstly, the speed of the acoustic waves becomes large, and secondly the right hand side goes to zero. However, it is crucial to check with such a condition that the critical value $\alpha_{\star}$ is not exceeded on the time step. We are not aware of a similar stability analysis for other numerical schemes dealing with this problem, but it is very likely that such a behavior is not specific to the kinetic scheme designed above. It might explain the difficulties reported concerning the close-packing limit [2, 7], [34, Sect. 3.2.1 \& 4].

Finally, we point out that, according to [6], the scheme can be shown to be energydecaying, under a suitable stability condition on the time step, the discrete energy being defined by

$$
\begin{aligned}
E^{k}= & \sum_{j=2}^{J} \frac{\delta x_{j}}{2} \alpha_{p, j}^{k}\left(u_{p, j}^{k}\right)^{2}+\sum_{j=1}^{J} \delta x_{j+1 / 2} \Phi\left(\alpha_{p, j+1 / 2}^{k}\right)+\sum_{j=2}^{J} \frac{\delta x_{j}}{2} \alpha_{f, j}^{k}\left(u_{f, j}^{k}\right)^{2} \\
& +\sum_{j=2}^{J}\left(\varrho_{p} \alpha_{p, j+1 / 2}+\varrho_{f} \alpha_{f, j+1 / 2}\right) \mathscr{G}\left(x_{j+1 / 2}\right)
\end{aligned}
$$

where the function $\Phi$ is defined in (14).

\section{$4 \quad$ Numerical Tests}

In this section, we present two numerical tests to illustrate the behaviour of the proposed scheme. As explained above, we are faced two distinct difficulties. Firstly, the pressure law $\alpha \mapsto \pi(\alpha)$ blows up as $\alpha \rightarrow \alpha_{\star}$ which induces local stiffness, and thus impacts the stability condition, as analyzed in Proposition 3.6. Secondly, we need to handle the constraint (19); it is equivalent to the divergence free condition $\nabla_{x} \cdot\left(\alpha_{p} u_{p}+\alpha_{f} u_{f}\right)=0$ and we wish to preserve this equivalence for the discrete quantities. We will discuss on numerical grounds the ability of our scheme in treating these difficulties. To this end, we start by disregarding the coupling, and we consider the system (20)-(21) only. It is nothing but the barotropic Euler system, with a singular pressure law. The test aims at illustrating the behaviour of the scheme when $\alpha_{p}$ gets closer to $\alpha_{\star}$. Next, we go back to the full model (15)-(18), and we simulate the sedimentation process of heavy particles in a fluid.

\subsection{Particles equations. A 1D Riemann problem}

We consider a Riemann problem for the (single phase) model (20)-(21) in the one dimensional case. The function $\pi$ is defined by

$$
\pi(\alpha)=\frac{\alpha^{\beta}}{\alpha_{\star}-\alpha},
$$

with $\alpha_{\star}=1$ and $\beta=2$. The constant $c^{2}$ is chosen equal to $\frac{(\beta-1)^{2}}{4 \beta}$ and the computational domain is $[-0.5,0.5]$. 


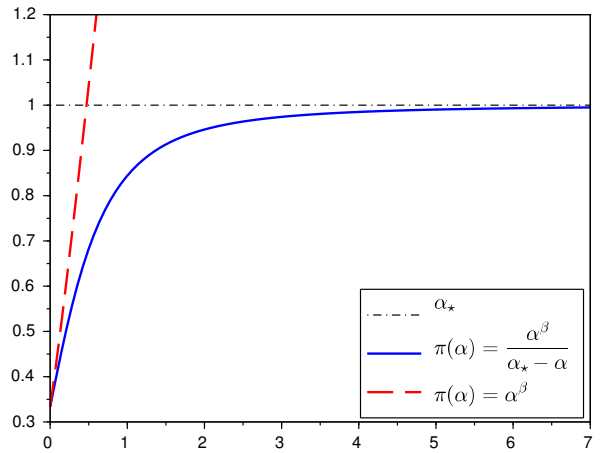

Figure 1: Intermediate volume fraction $\alpha_{\mathrm{M}}$ as a function of the velocity $u$

The initial data is made of two constant states $\left(\alpha_{\mathrm{L}}, u_{\mathrm{L}}\right)=(1 / 3, u)$ and $\left(\alpha_{\mathrm{R}}, u_{\mathrm{R}}\right)=$ $(1 / 3,-u)(u>0)$ with a discontinuity located at $x=0$. We use Neumann like boundary conditions but the simulation is stopped before the solution may interact with the boundary, so that we can consider that we compute the solution of the problem set on the whole line.

For such initial data, the structure of the solution is well known. It is made of three constant states (the two initial states $\left(\alpha_{\mathrm{L}}, u_{\mathrm{L}}\right)$ and $\left(\alpha_{\mathrm{R}}, u_{\mathrm{R}}\right)$, and an additional intermediate state $\left.\left(\alpha_{\mathrm{M}}, u_{\mathrm{M}}\right)\right)$. The value of $u_{\mathrm{M}}$ is 0 whereas the value $\alpha_{\mathrm{M}}$ depends on $u\left(=\left|u_{\mathrm{L}}\right|=\left|u_{\mathrm{R}}\right|\right)$. The behaviour of $\alpha_{\mathrm{M}}$ as a function of $u$ is given on Figure 1 (solid line). The dotted line shows the behaviour of $\alpha_{\mathrm{M}}$ if we use the perfect gas state law $\pi(\alpha)=\alpha^{\beta}$ instead of the close-packing term. In both case, $\alpha_{\mathrm{M}}$ increases with $u$ but, as expected, the close-packing term prevents $\alpha_{\mathrm{M}}$ to be greater than $\alpha_{\star}$.

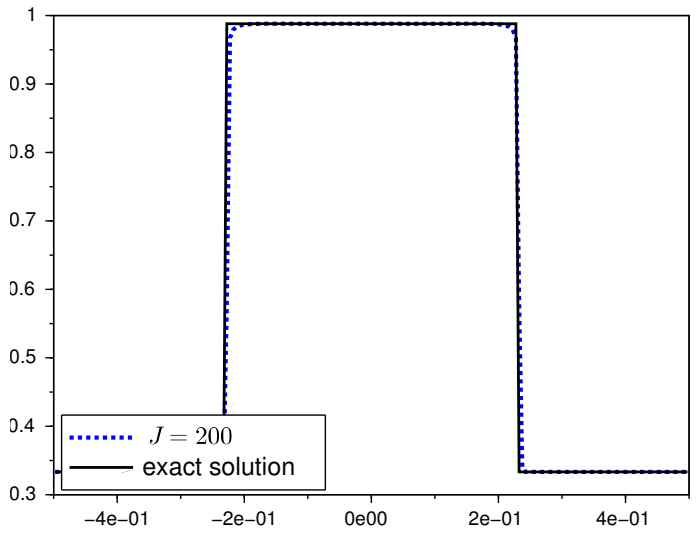

(a) Volume fraction

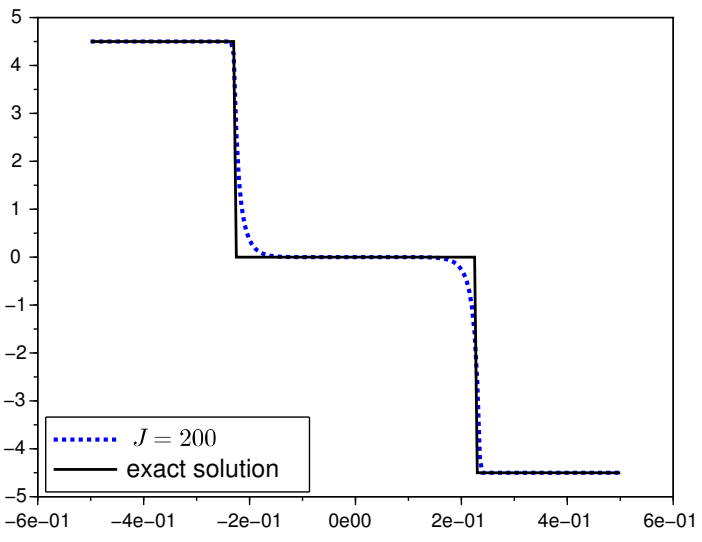

(b) Velocity

Figure 2: Exact and numerical $\left(J=200, \delta t=4.04410^{-6}\right)$ solution at $t=0.1$ with $u=4.5$

The constant states $\left(\alpha_{\mathrm{L}}, u_{\mathrm{L}}\right),\left(\alpha_{\mathrm{R}}, u_{\mathrm{R}}\right)$ and $\left(\alpha_{\mathrm{M}}, u_{\mathrm{M}}\right)$ are linked by two shock waves going away from $x=0$. An example of simulation is given in Figure 2. The numerical solution is plotted with dotted lines whereas the exact solution is plotted with a solid line. We observe that the scheme behaves very well, the values of intermediate states 
and the speed of shocks are correctly computed. Further examples on barotropic Euler equations are presented in [6].

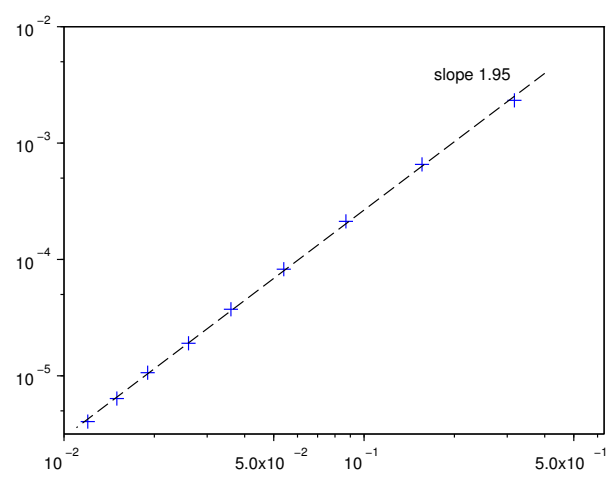

Figure 3: Maximal "acceptable" time step $\delta t$ as a function of $1-\frac{\alpha_{\mathrm{M}}}{\alpha_{\star}}$

We end this section by illustrating the result of Proposition 3.6. The mesh size is fixed $(J=200)$ and we perform several simulations for different values of $u \in$ $\{0.5,1,1.5,2,2.5,3,3.5,4,4.5\}$ and different values of the time step $\delta t$. For each values of $u$, we select the greater value of $\delta t$ which yields an "acceptable" result (in the sense that it raises no oscillation at $t=0.1$ ). In Figure 3, we plot this selected time step as a function of $1-\frac{\alpha_{\mathrm{M}}}{\alpha_{\star}}$. In a logarithmic scale, we obtain a straight line with a slope of 2 . It is consistent with the relation (40) since when $\alpha$ becomes close to $\alpha_{\star}$, the characteristic velocities behave like $\left(1-\frac{\alpha_{\mathrm{M}}}{\alpha_{\star}}\right)^{-1}$. This discussion is far from harmless. Indeed, many references report a lack of robustness of the numerical methods with respect to the close-packing threshold, see for instance the comments in $[2,7,34]$. However, we are not aware of a similar stability analysis, and we realize that (40), confirmed by this simple example, is by far more demanding than the CFL condition that guarantees the positivity of the density.

\subsection{Sedimentation of heavy particles}

In this section, we simulate the sedimentation process of heavy particles in a fluid using the complete model (15)-(19). The test case is inspired from [1, 7, 41]. Particles are immersed in a fluid which is enclosed in a box. At initial time, the particles are distributed in the whole domain $\left(\alpha_{p}(t=0, \cdot)=\alpha_{f}(t=0, \cdot)=0.5\right)$ and the system is at rest $\left(u_{p}(t=0, \cdot)=u_{f}(t=0, \cdot)=0\right)$. The only external force term is the gravity which is purely vertical. Hence, due to the symmetry of the initial conditions, the flow remains purely vertical during the whole time evolution. The simulations can be performed in the one dimensional framework following the vertical direction (see the first frame of Figure 6).

In the simulations above, the values of the physical parameters of the model are chosen as follows:

$$
\varrho_{f}=1, \quad \varrho_{p}=10^{3}, \quad g=-10, \quad \mu=10^{-4} .
$$


We perform simulations with friction using $D_{p}=\frac{9}{2} \frac{\mu}{\varrho_{p} a^{2}}, a=10^{-3}$ or without friction using $D_{p}=0$. The close-packing term is defined by

$$
\pi\left(\alpha_{p}\right)=\frac{\alpha_{p}{ }^{\beta}}{\alpha_{\star}-\alpha_{p}}, \quad \text { with } \quad \alpha_{\star}=0.7, \beta>1
$$

We first describe, in Section 4.2.1, the influence of parameters $\beta$ and $c$ (which define the amplitude of the close packing term) on the profile at equilibrium (steady solution with zero velocities). Then, we present numerical results using $c=1, \beta=2$ and $c=0.2, \beta=4$ in Section 4.2.2.

We use different mesh sizes from $J=100$ to $J=200$ and, when it is not explicitly specified (as in the convergence study), the (variable) time step is automatically set at each time iteration with the definition:

$$
\delta t=\min \left(\delta t_{1}, \delta t_{2}\right) \text { with }\left\{\begin{array}{l}
\delta t_{1}=0.9 \frac{\min _{j}\left(\delta x_{j+1 / 2}\right)}{\max _{j}\left(\left|u_{f, j}\right|\right)}, \\
\delta t_{2}=0.9 \min _{j}\left(\delta x_{j+1 / 2}\right) \frac{1-\max _{j}\left(\alpha_{p, j+1 / 2}\right) / \alpha_{\star}}{\max _{j}\left(c\left(\alpha_{p, j+1 / 2}\right)\right)+\max _{j}\left(\left|u_{p, j}\right|\right)},
\end{array}\right.
$$

which is derived from the stability conditions (38), (39) and (40).

\subsubsection{Equilibrium states}

We numerically observe that, for large time, the system converges toward an equilibrium with zero velocity. At the continuous level, the equilibrium profile $\alpha_{p, \infty}$ satisfies:

$$
\alpha_{p, \infty} \geq 0, \quad c^{2} \partial_{x}\left(\pi\left(\alpha_{p, \infty}\right)\right)=\alpha_{p, \infty} \bar{g}, \quad \text { and } \quad \int_{\Omega} \alpha_{p, \infty}(x) \mathrm{d} x=\mathscr{V}_{0},
$$

where $\bar{g}=g\left(1-\varrho_{f} / \varrho_{p}\right)$ and $\mathscr{V}_{0}=\int_{\Omega} \alpha_{p}(t=0, x) \mathrm{d} x$ is the volume occupied by the particles at the initial time. This problem admits a unique continuous solution. Since $\pi^{\prime}(0)=0$ and $\pi^{\prime}(\alpha)>0, \forall \alpha \in\left(0, \alpha_{\star}\right)$, there exists $x_{0} \in \Omega=(a, b)$ such that $\alpha_{p, \infty}$ is a positive decreasing function of class $\mathscr{C}^{1}$ on $\left(a, x_{0}\right)$ and vanishes on $\left[x_{0}, b\right)$. The value of $\alpha_{p, \infty}$ at $a$ is given by

$$
\alpha_{p, \infty}(a)=\pi^{-1}\left(-\frac{\bar{g}}{c^{2}} \mathscr{V}_{0}\right),
$$

and its expression on $\left(a, x_{0}\right)$ is given by

$$
\alpha_{p, \infty}(x)=f_{\beta}^{-1}\left(\frac{\bar{g}}{c^{2}}(x-a)+f_{\beta}\left(\alpha_{p, \infty}(a)\right)\right), \forall x \in\left(a, x_{0}\right),
$$

where $f_{\beta}$ stands for a primitive of the function $\alpha \mapsto \pi^{\prime}(\alpha) / \alpha$. In the case where $\beta$ is an integer, the functions $f_{\beta}$ can be (recursively) defined as follows

$$
\left\{\begin{array}{l}
f_{\beta+1}(\alpha)=\alpha_{\star} f_{\beta}(\alpha)-\frac{\beta}{\beta-1} \alpha^{\beta-1}, \quad \forall \beta \geq 2, \forall \alpha \in\left[0, \alpha_{\star}\right), \\
f_{2}(\alpha)=\frac{\alpha_{\star}}{\alpha_{\star}-\alpha}-\ln \left(\alpha_{\star}-\alpha\right), \quad \forall \alpha \in\left[0, \alpha_{\star}\right) .
\end{array}\right.
$$


With these functions at hand, we can also give the expression of $x_{0}$

$$
x_{0}=\min \left(b, a+\frac{c^{2}}{\bar{g}}\left(f_{\beta}(0)-f_{\beta}\left(\alpha_{p, \infty}(a)\right)\right)\right) .
$$

The relations (41) and (42) allow us to numerically compute the equilibrium profile $\alpha_{p, \infty}$ up to the machine epsilon using a Newton method at each point.

We first examine the influence of the different parameters of the model on the shape of the equilibrium profile. The role of $\alpha_{\star}$ is clear: it is a threshold imposed on the volume fraction but the influence of $c$ and $\beta$ deserves to be illustrated. Figure $4 \mathrm{a}$ presents the equilibrium profiles obtained for different values of $c \in\{0.1,0.4,0.7,1\}$ and $\beta=2$ while Figure $4 \mathrm{~b}$ presents the equilibrium profiles obtained for different values of $\beta \in\{2,3,4,5\}$ and $c=1$. The smaller the value of $c$, the stiffer the equilibrium profile. Similarly as $c$ decreases, the value $\alpha_{p, \infty}(a)$ gets closer to $\alpha_{\star}$. Similar conclusion apply when, $c$ being fixed, we make $\beta$ increase. In [3], the authors recommend to use $2 \leq \beta \leq 5$ and, in [41], the authors use $\beta=3$ and $c \sim 0.2$.

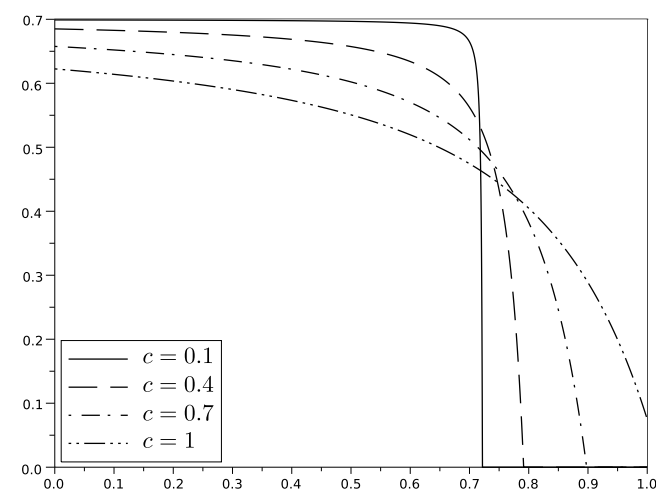

(a) Influence of $c, \beta=2$

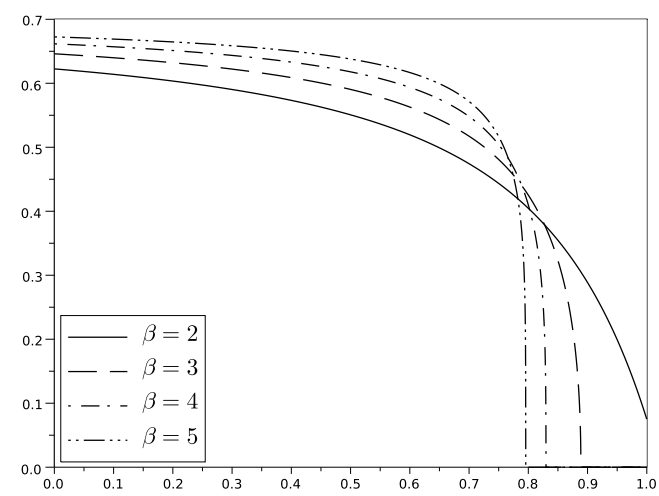

(b) Influence of $\beta, c=1$

Figure 4: Influence of model parameters on the shape of the equilibrium profile.

As time becomes large we expect that the solutions of the complete model (15)(19) tend to the equilibrium profile with zero velocities. We are going to check this behaviour on numerical grounds. Simulations are performed with $J=100$. The results are presented on Figure $5 \mathrm{a}(c=1, \beta=2)$, Figure $5 \mathrm{~b}(c=0.4, \beta=2)$ and Figure $5 \mathrm{c}$ $(c=0.2, \beta=4)$. The profiles computed with the relations (41) and (42) and large time simulations agree very well. The scheme does not produce any spurious oscillations in the vacuum regions that appear when $c$ is small. However for small $c$, the value of the particles density is very close to the close-packing threshold $\alpha_{\star}$ and therefore the time step is very constrained by the stability conditions (38), (39) and (40), see Figure 12. The simulations for $c$ small or $\beta$ large are more challenging. We can observe that the particles velocity at final time is not exactly zero, instead it remains of order $\delta x$ since we do not use an elaborate well-balanced expression for the gravity term; we refer the reader to [9] for an introduction to these techniques. 


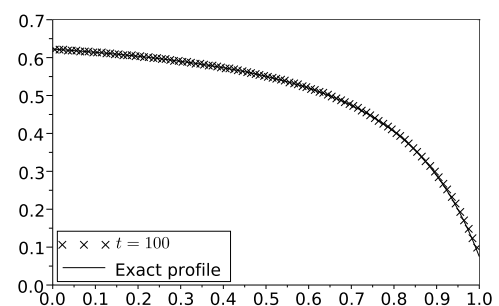

(a) $c=1$ and $\beta=2$

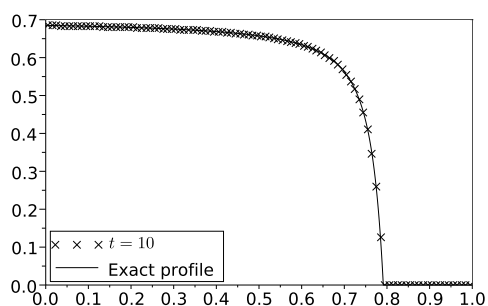

(b) $c=0.4$ and $\beta=2$

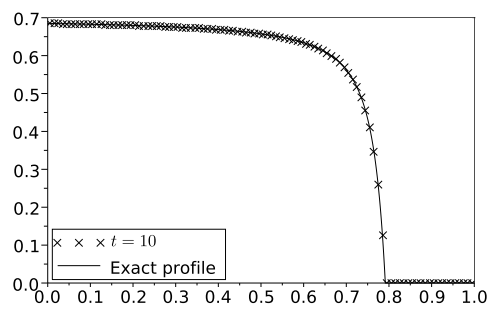

(c) $c=0.2$ and $\beta=4$

Figure 5: Convergence to the equilibrium profile for large time simulations $(J=100)$.

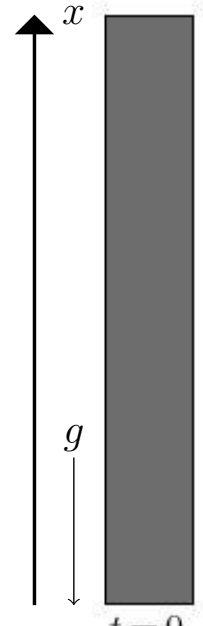

$t=0$

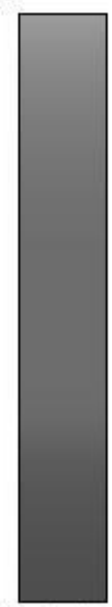

0.1

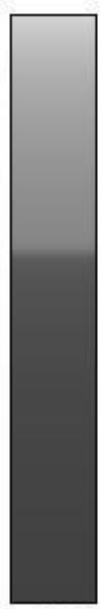

0.2

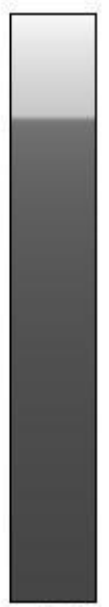

0.3

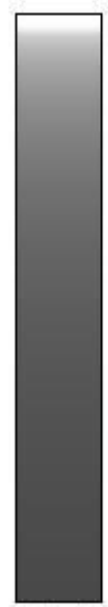

0.4

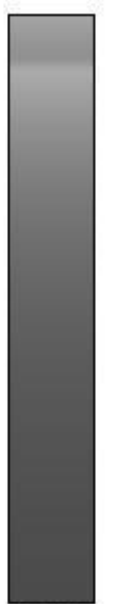

0.5

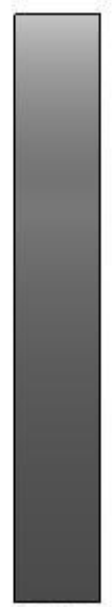

0.6

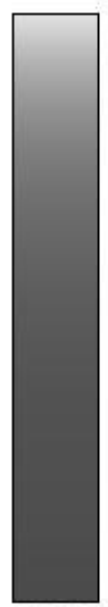

0.7

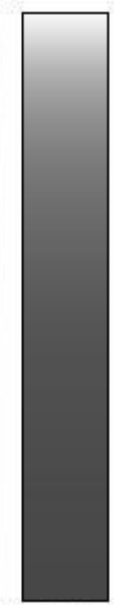

0.8

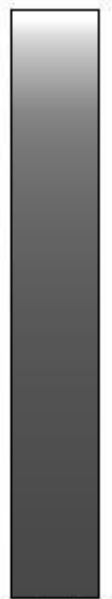

$0.9 \quad 1$

Figure 6: Time evolution of the particle volume fraction using a grayscale representation $\left(J=200, \delta t=210^{-5}\right.$ with friction).

\subsubsection{Time evolution of numerical solutions}

We first perform simulations using $\beta=2$ and $c=1$. The time evolution of the system with friction is presented on Figure 6 using a grayscale representation. Due to gravity, the heavy particles first move downward while the fluid moves upward. Most of the particles remain packed at the bottom of the box but we observe at time 0.2 that some particles have bounced back and move upward until time 0.5 when they reach the top of the box. A new bounce occurs and so on...

The results we present here are very well converged in time. This is illustrated on Figure 7 where the result obtained at time $t=0.5$ with different time steps $\left(\delta t=10^{-4}\right.$, $\delta t=5 \times 10^{-5}$ and $\left.\delta t=2 \times 10^{-5}\right)$ and a fixed mesh size $(J=100)$ are compared. The three curves are very close, this is not surprinsing since we have seen, in Section 3.2.3 (see also numerical illustration in Section 4.1), that the stability condition (40) compels to use small time steps. The convergence study in space is presented in Figure 8. The 


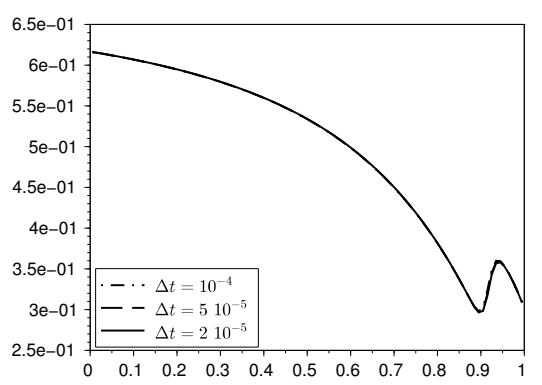

(a) Particles volume fraction $\alpha_{p}$

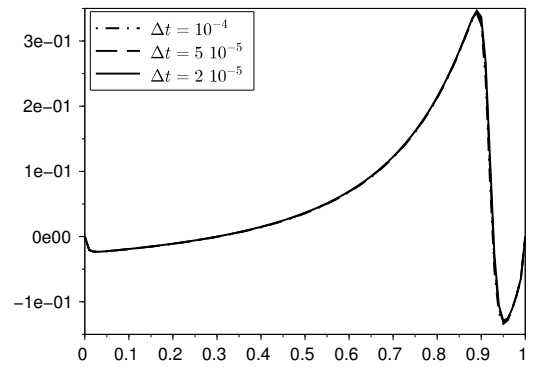

(b) Particles velocity $u_{p}$

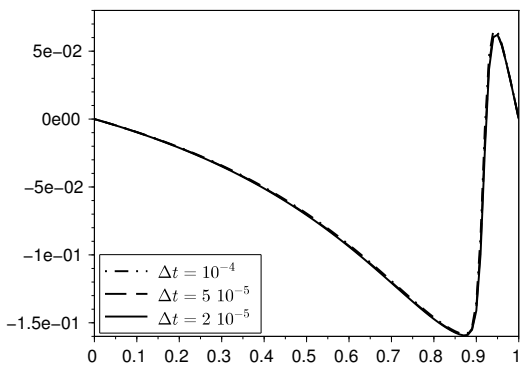

(c) Fluid velocity $u_{f}$

Figure 7: Time convergence study at time $t=0.5$ using a fixed mesh size $(J=100)$

result obtained at time $t=0.5$ with different mesh sizes $(J=100, J=150$ and $J=200)$ and a fixed time step $\left(\delta t=2 \times 10^{-5}\right)$ are compared. With $J=200$, the result appears to be rather well converged.

In Figure 9, 10 and 11, the results obtained (using $\delta t=2 \times 10^{-5}$ and $J=200$ ) with or without friction are compared during the time evolution. Figure 9 presents the evolution of the particles volume fraction; Figure 10 the evolution of the particle velocity and Figure 11 the evolution of the fluid velocity. The behaviour of the system is globally the same in the two cases. As mentionned above, the particles first move downward while the fluid moves upward. We indeed observe at time $t=0.1$ that (i) the particle volume fraction has increased in the bottom part of the domain $(x<0.4)$ while it has decreased in the top of the domain $(x>0.6)$ and (ii) the particle velocity is non positive whereas the fluid velocity is non negative. Then, at time $t \sim 0.2$, a bounce of some particles at the bottom of the domain is observed since the particles velocity becomes positive. The particles go back until $t \sim 0.4$ where a new bounce occurs at the top of the domain. As expected, we observe that the presence of the friction term slightly damps the motion of particles. We also remark that the volume fraction of the particles do not exceed $\alpha_{\star}$ during the whole time evolution.

To end this section, we turn to a more challenging simulation using $c=0.2$ and $\beta=4$. The time evolution of the time step in presented in Figure 12. The time evolution of the particles volume fraction is presented in Figure 13. The heavy particles move downward while the fluid moves upward. The particles are rapidly packed at the bottom of the box and contrarily to the case with $c=1$ and $\beta=2$, we do not observe any bounce at the bottom or at the top of the box. 


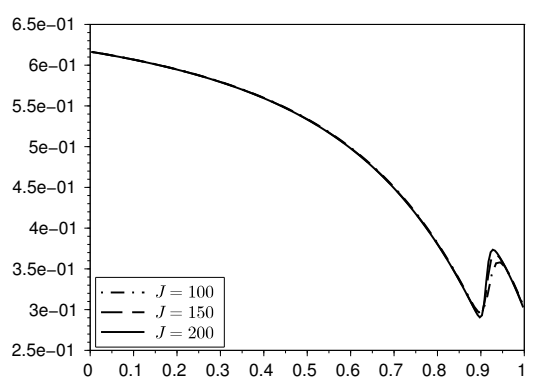

(a) Particles volume fraction $\alpha_{p}$

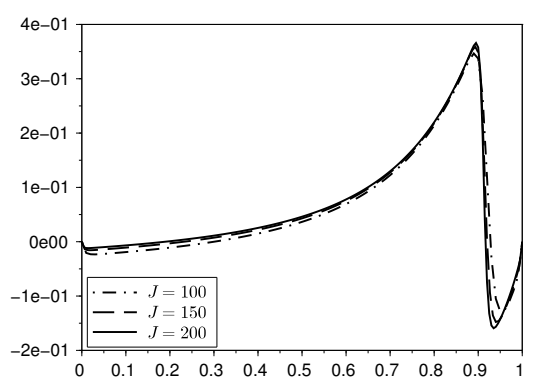

(b) Particles velocity $u_{p}$

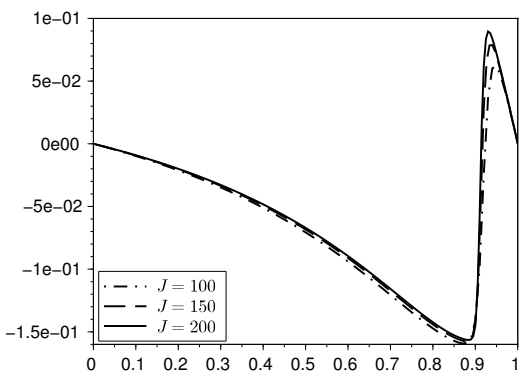

(c) Fluid velocity $u_{f}$

Figure 8: Space convergence study at time $t=0.5$ using a fixed time step $\left(\delta t=210^{-5}\right)$

\section{A Energy dissipation: Proof of Proposition 2.3}

We first multiply the equations for particles (9) by $\varrho_{p} \frac{|\xi|^{2}}{2}$ and integrate with respect to $\xi$. An integration by parts of the forcing terms yields (assuming that $f$ vanishes for large $|\xi|)$

$$
\begin{aligned}
\partial_{t}\left(\varrho_{p} \int \frac{|\xi|^{2}}{2} f \mathrm{~d} \xi\right)+\nabla_{x} \cdot\left(\varrho_{p} \int \xi \frac{|\xi|^{2}}{2} f \mathrm{~d} \xi\right) \\
\quad=-\int \xi \cdot \varrho_{p} D_{p}\left(\xi-u_{f}\right) f \mathrm{~d} \xi-\left(\frac{P_{\star}}{\alpha_{p}} \nabla_{x} \pi\left(\alpha_{p}\right)-\varrho_{p} g+\nabla_{x} P\right) \cdot \int \xi f \mathrm{~d} \xi .
\end{aligned}
$$

Classicaly, we then multiply the fluid momentum balance equation (8) by $\varrho_{f} u_{f}$ and, using the mass balance equation (7), we obtain

$$
\begin{aligned}
& \partial_{t}\left(\frac{\alpha_{f} \varrho_{f}\left|u_{f}\right|^{2}}{2}\right)+\nabla_{x} \cdot\left(u_{f} \frac{\alpha_{f} \varrho_{f}\left|u_{f}\right|^{2}}{2}\right) \\
& =u_{f} \cdot\left(\alpha_{f} \varrho_{f} g+\frac{4}{3} \pi \bar{a}^{3} \varrho_{p} \int D_{p}\left(\xi-u_{f}\right) f \mathrm{~d} \xi-\alpha_{f} \nabla_{x} P+\nabla_{x} \cdot\left(\alpha_{f} \tau\right)\right) .
\end{aligned}
$$



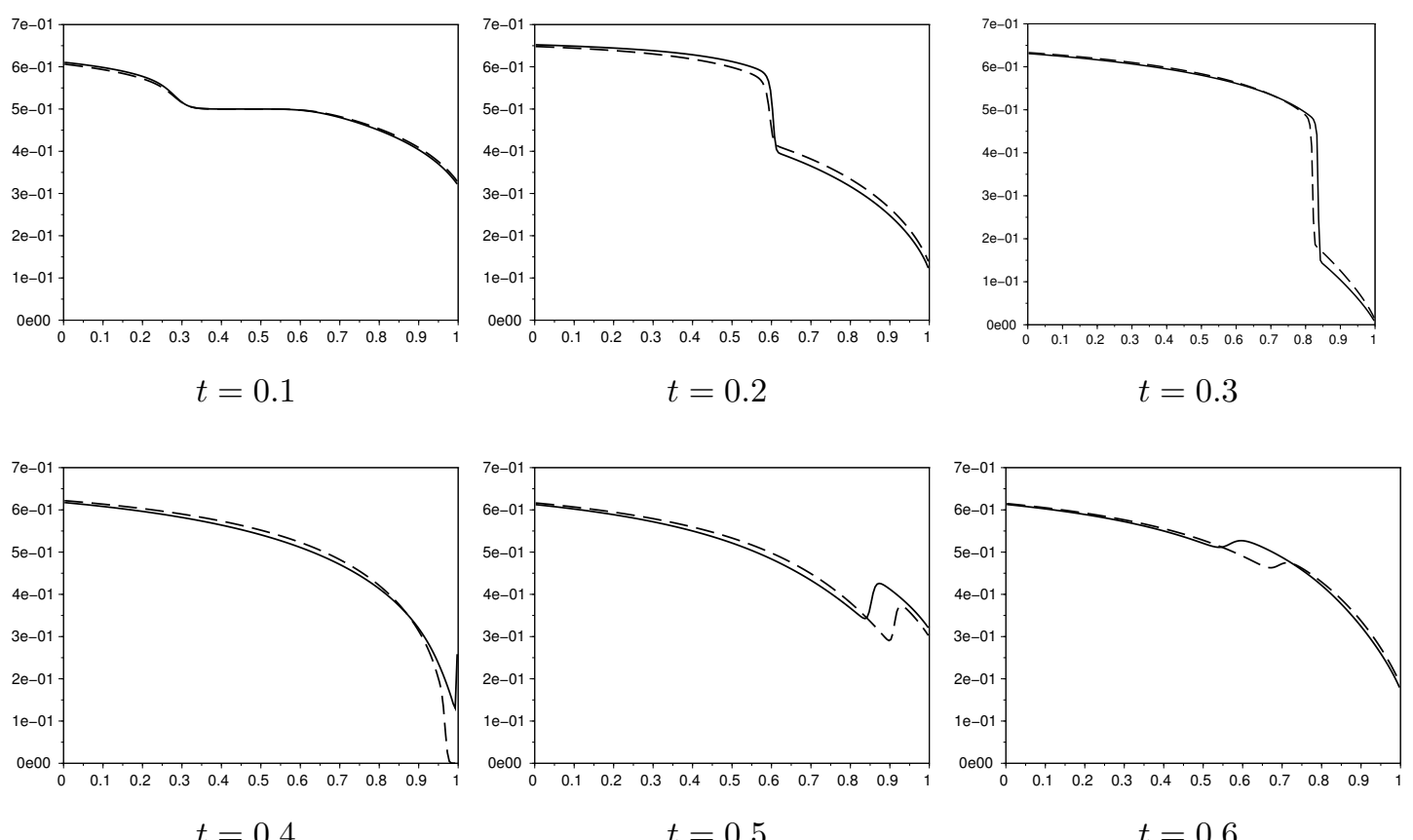

$t=0.4$

$t=0.5$

$t=0.6$
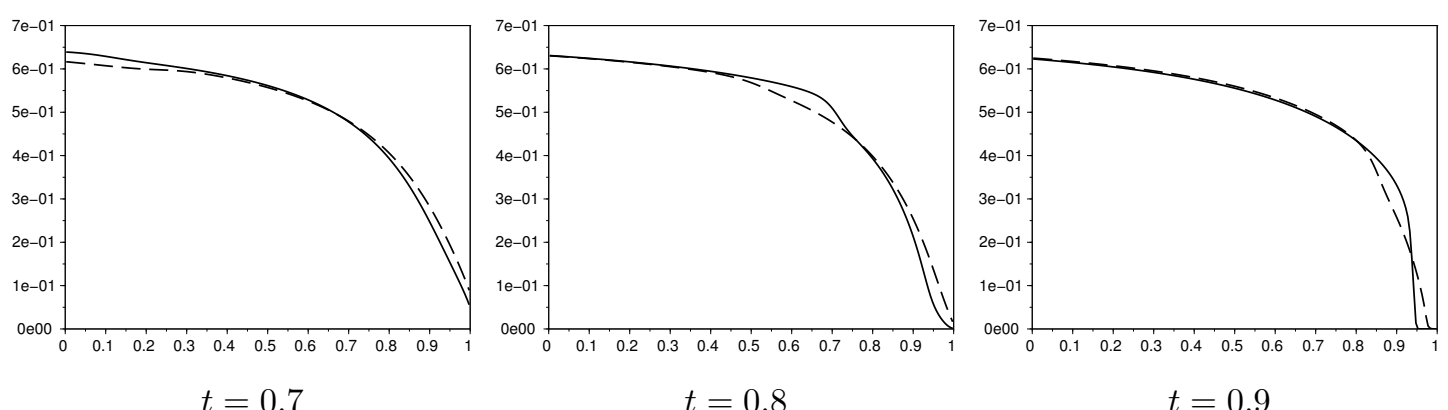

$t=0.8$

$t=0.9$

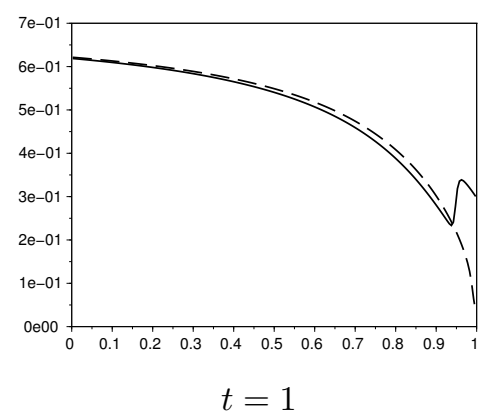

Figure 9: Time evolution of particles volume fraction $\alpha_{p}$. Comparison of results obtained with (dotted lines) and without (solid lines) friction 

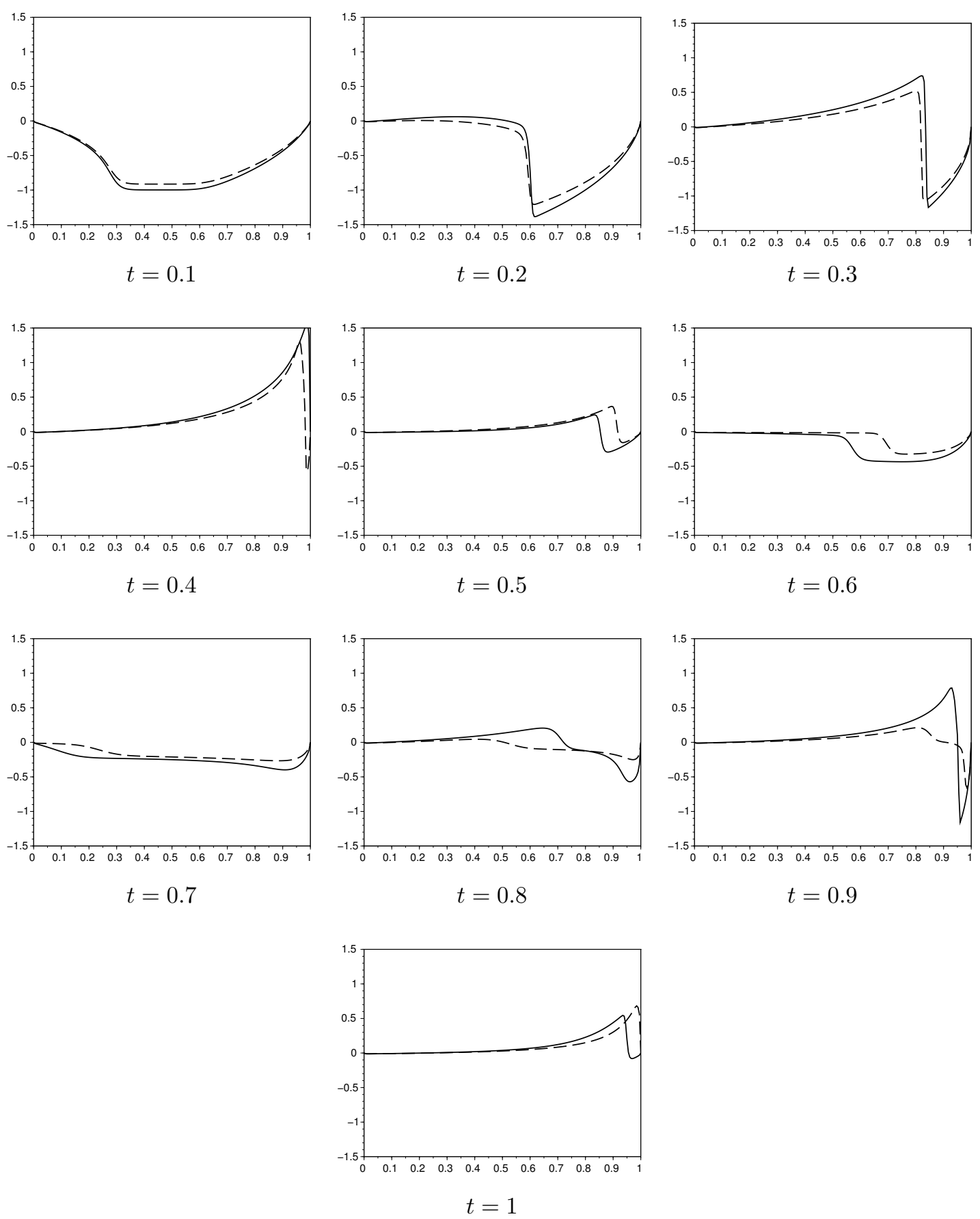

Figure 10: Time evolution of particles velocity $u_{p}$. Comparison of results obtained with (dotted lines) and without (solid lines) friction 

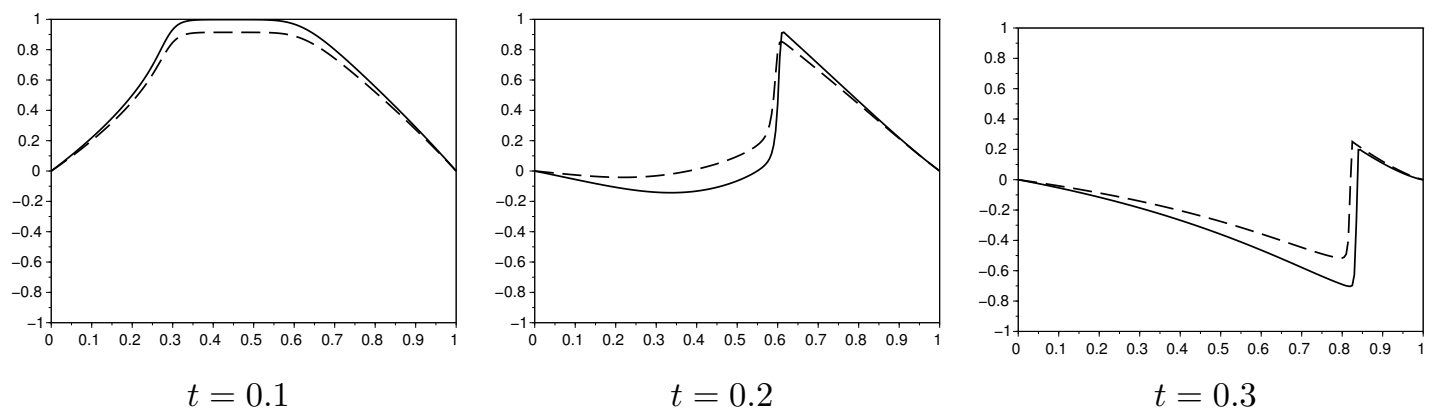

$t=0.2$

$t=0.3$
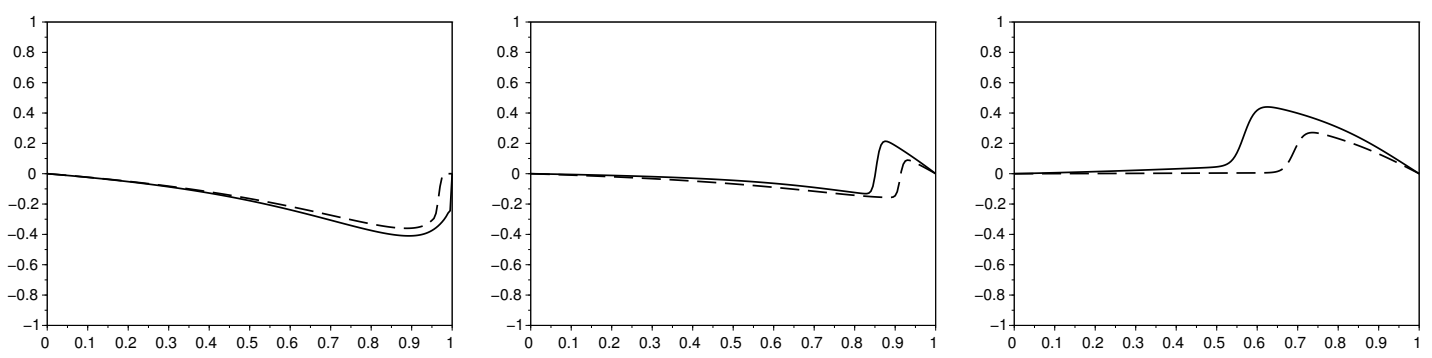

$t=0.4$

$t=0.5$

$t=0.6$
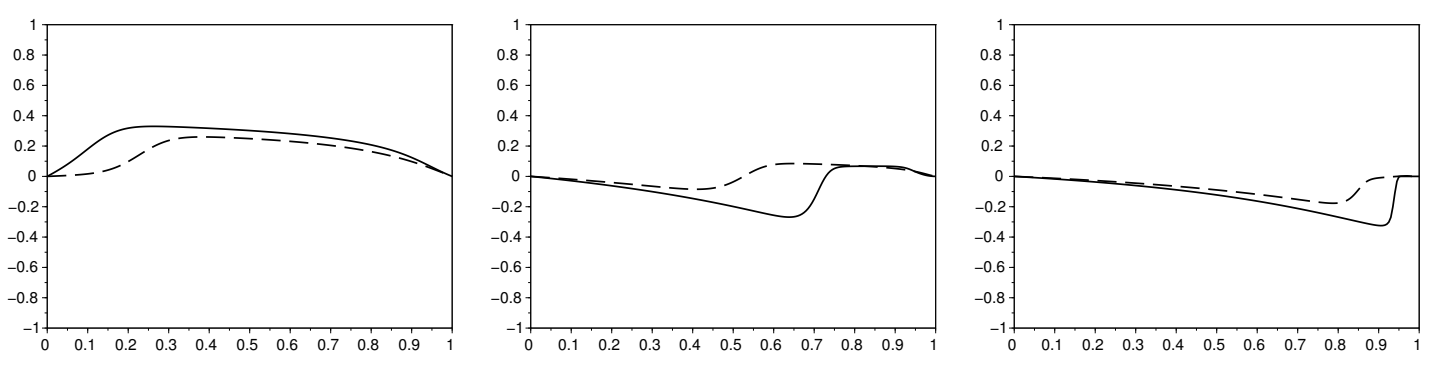

$t=0.7$

$t=0.8$

$t=0.9$

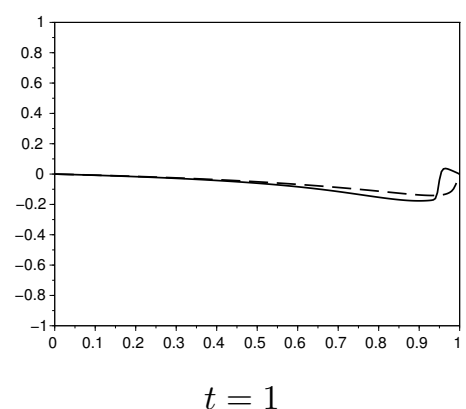

Figure 11: Time evolution of the fluid velocity $u_{f}$. Comparison of results obtained with (dotted lines) and without (solid lines) friction 


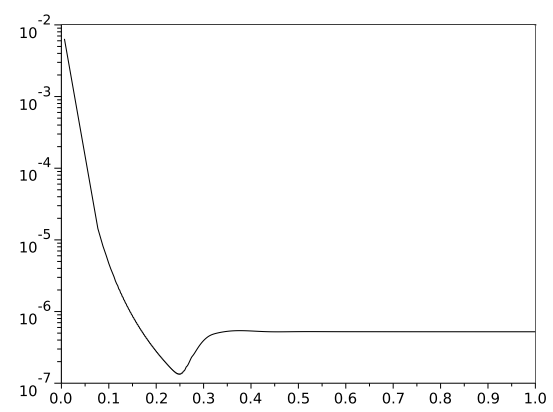

Figure 12: Time steps as a function of time

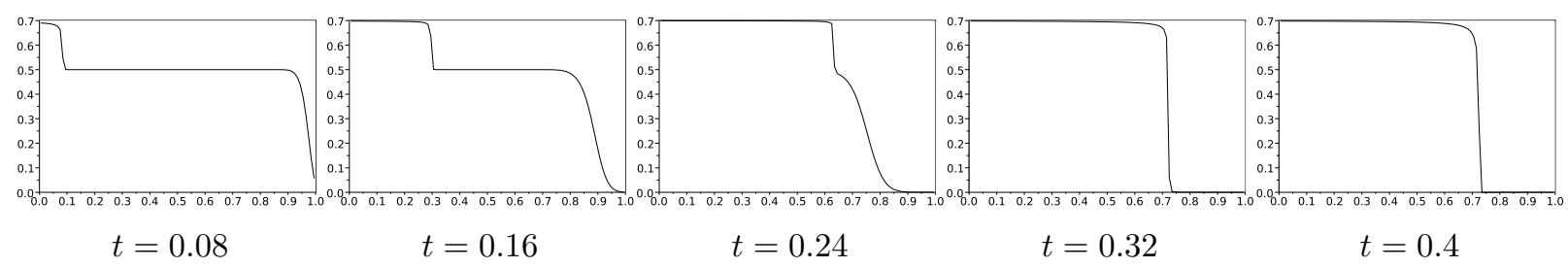

Figure 13: Time evolution of particles volume fraction $\alpha_{p}$

Since $\frac{4}{3} \pi \bar{a}^{3} \int \xi f \mathrm{~d} \xi=\alpha_{p} u_{p}$, by combining (43) and (44), we readily find

$$
\begin{aligned}
& \partial_{t}\left(\frac{4}{3} \pi \bar{a}^{3} \varrho_{p} \int \frac{|\xi|^{2}}{2} f \mathrm{~d} \xi+\frac{\alpha_{f} \varrho_{f}\left|u_{f}\right|^{2}}{2}\right) \\
&+\nabla_{x} \cdot\left(\frac{4}{3} \pi \bar{a}^{3} \varrho_{p} \int \xi \frac{|\xi|^{2}}{2} f \mathrm{~d} \xi+u_{f} \frac{\alpha_{f} \varrho_{f}\left|u_{f}\right|^{2}}{2}\right)+\frac{4}{3} \pi \bar{a}^{3} \varrho_{p} \int D_{p}\left(\xi-u_{f}\right)^{2} f \mathrm{~d} \xi \\
&=-\nabla_{x} P \cdot\left(\alpha_{f} u_{f}+\alpha_{p} u_{p}\right)+\left(\varrho_{f} \alpha_{f} u_{f}+\varrho_{p} \alpha_{p} u_{p}\right) \cdot g \\
&-P_{\star} u_{p} \cdot \nabla_{x} \pi\left(\alpha_{p}\right)-u_{f} \cdot \nabla_{x} \cdot\left(\alpha_{f} \tau\right) .
\end{aligned}
$$

It now remains to deal with all the forcing terms in the right-hand side of (50):

- pressure terms: Summing the mass balance equations (7) and (11), and using the volume constraint (10), we get that the mean volume velocity is divergence-free:

$$
\nabla_{x} \cdot\left(\alpha_{f} u_{f}+\alpha_{p} u_{p}\right)=0
$$

and, consequently

$$
\nabla_{x} P \cdot\left(\alpha_{f} u_{f}+\alpha_{p} u_{p}\right)=\nabla_{x} \cdot\left(P\left(\alpha_{f} u_{f}+\alpha_{p} u_{p}\right)\right) .
$$

- gravity terms: Using the definition (13) of the gravity potential $\mathscr{G}$ and the mass balance equations (7) and (11), we have

$$
g \cdot\left(\varrho_{p} \alpha_{p} u_{p}+\varrho_{f} \alpha_{f} u_{f}\right)=-\nabla_{x} \cdot\left(\mathscr{G}\left(\varrho_{p} \alpha_{p} u_{p}+\varrho_{f} \alpha_{f} u_{f}\right)\right)-\mathscr{G} \partial_{t}\left(\varrho_{p} \alpha_{p}+\varrho_{f} \alpha_{f}\right) .
$$


- close-packing term: We compute

$$
\partial_{t} \Phi\left(\alpha_{p}\right)+\nabla_{x} \cdot\left(\Phi\left(\alpha_{p}\right) u_{p}\right)=\Phi^{\prime}\left(\alpha_{p}\right)\left(\partial_{t} \alpha_{p}+u_{p} \cdot \nabla_{x} \alpha_{p}\right)+\Phi\left(\alpha_{p}\right) \nabla_{x} \cdot u_{p}
$$

Hence, by using the mass balance equation (11), we find

$$
\partial_{t} \Phi\left(\alpha_{p}\right)+\nabla_{x} \cdot\left(\Phi\left(\alpha_{p}\right) u_{p}\right)=\left(-\alpha_{p} \Phi^{\prime}\left(\alpha_{p}\right)+\Phi\left(\alpha_{p}\right)\right) \nabla_{x} \cdot u_{p}
$$

Bearing in mind the relation (14) (ii), we finally get

$$
\partial_{t} \Phi\left(\alpha_{p}\right)+\nabla_{x} \cdot\left(\Phi\left(\alpha_{p}\right) u_{p}+c^{2} \pi\left(\alpha_{p}\right) u_{p}\right)=c^{2} u_{p} \cdot \nabla_{x} \pi\left(\alpha_{p}\right)
$$

- viscosity terms: We first observe that

$$
u_{f} \cdot \nabla_{x}\left(\alpha_{f} \tau\right)=\nabla_{x}\left(\alpha_{f} \tau . u_{f}\right)-\alpha_{f} \tau: \nabla_{x} u_{f},
$$

where $A: B=\sum_{i, j} A_{i j} B_{i j}$ stands for the contracted product of the two matrix $A$ and $B$. Then, the equality

$$
\nabla_{x} u_{f}: \tau=\frac{\mu}{2}\left|\nabla_{x} u_{f}+\left(\nabla_{x} u_{f}\right)^{\top}\right|^{2}-\frac{2 \mu}{3}\left|\nabla_{x} \cdot u_{f}\right|^{2}
$$

comes from the definition (1) of $\tau$

$$
\tau=\mu\left(\nabla_{x} u_{f}+\left(\nabla_{x} u_{f}\right)^{\top}-\frac{2}{3} \operatorname{Tr}\left(\nabla_{x} u_{f}\right) \mathrm{Id}\right),
$$

and the two straightforward following properties of the contracted product

$$
A: \operatorname{Id}=\operatorname{Tr}(A), \quad \text { and } \quad A: S=A^{\top}: S \quad \text { if } S \text { is a symmetric tensor, }
$$

where $\operatorname{Tr}(A)=\sum_{i} A_{i i}$ stands for the trace of the matrix $A$.

We conclude by inserting (47), (48), (49), (50) and (51) into (45).

The proof of Proposition 2.4 follows exactly from the same manipulations we did for the kinetic model, the counterpart (43) being obtained by multiplying the particle momentum balance equation (16) by $\varrho_{p} u_{p}$ and using the mass balance equation (15) to transform the inertia terms.

We end this section by noting that the left hand side of the equality (51) is non negative (in dimension $N=3$ ). It comes from the fact that the quadratic form $q(S)=$ $2 \mu\left(S:\left(S-\frac{1}{3} \operatorname{Tr}(S) \mathrm{Id}\right)\right)$ is positive on the set of symmetric tensors. It can be readily verified since it reduces to the study of a quadratic form in $\mathbb{R}^{N}$ by diagonalizing the symmetric matrices $S$ and noting that $q\left(P^{\top} D P\right)=q(D)$ for all matrix $D$ and for all orthogonal matrix $P$. The conclusion is obtained by observing that

$$
\nabla_{x} u_{f}: \tau=q\left(\frac{\nabla_{x} u_{f}+\left(\nabla_{x} u_{f}\right)^{\top}}{2}\right)
$$




\section{B Proof of Proposition 3.4}

We justify the existence of solutions to the non linear problem (37) by applying the topological degree theory, see [20] (in finite dimension).

Lemma B.1 (Topological degree) Let $W$ be a finite dimensional real vector space, endowed with the norm $|\cdot|_{W}$, and $\mathrm{G}$ a continuous function from $W$ to $W$. Assume that there exists a continuous function $\mathrm{H}$ from $W \times[0 ; 1]$ to $W$ satisfying

- $\mathrm{H}(\cdot, 1)=\mathrm{G}$ and $\mathrm{H}(\cdot, 0)$ is affine,

- $\exists R>0$ such that $\forall(w, \delta) \in W \times[0 ; 1]$, if $\mathrm{H}(w, \delta)=0$ then $|w|_{W} \neq R$,

- the equation $\mathrm{H}(w, 0)=0$ has a solution $w \in W$ such that $|w|_{W}<R$.

Then there exists at least one solution $w \in W$ such that $\mathrm{G}(w)=0$ and $|w|_{W}<R$.

Here, we consider

$$
W=\left\{\left(\Phi_{j+1 / 2}\right)_{j=1, . ., J} \in \mathbb{R}^{J}, \sum_{j=1}^{J} \Phi_{j+1 / 2}=0\right\}
$$

endowed with following norm

$$
|w|_{W}^{2}=\sum_{j=2}^{J} \delta x_{j+1 / 2}\left(\nabla_{j}^{D} \Phi\right)^{2}, \quad \forall w=\left(\Phi_{j+1 / 2}\right)_{j=1, . ., J} \in W .
$$

The quantities $\alpha_{p}^{k+1}, \alpha_{f}^{k+1}, \widetilde{u_{p}}$ and $\widetilde{u_{f}}$ are given, and, for the the sake of notational simplicity, we skip the superscripts $\bullet^{k+1}$ and $\bullet$ throughout the proof. For $w=$ $\left(\Phi_{j+1 / 2}\right)_{j=1, . ., J} \in W$ and $\delta \in[0,1]$, we set

$$
H(w, \delta)=\mathbb{D}^{(1) \delta}\left(\alpha_{p}, u_{p}-\frac{\delta t}{\varrho_{p}} \nabla^{D} \Phi\right)+\mathbb{D}^{(2) \delta}\left(\alpha_{f}, u_{f}-\frac{\delta t}{\varrho_{f}} \nabla^{D} \Phi\right)
$$

where

$$
\left[\mathbb{D}^{(1) \delta}\left(\alpha_{p}, u_{p}-\frac{\delta t}{\varrho_{p}} \nabla^{D} \Phi\right)\right]_{j+1 / 2}=\frac{\mathscr{F}_{j+1}^{\delta}-\mathscr{F}_{j}^{\delta}}{\delta x_{j+1 / 2}}, \quad j=1, . ., J,
$$

with $\mathscr{F}_{1}^{\delta}=0=\mathscr{F}_{J+1}^{\delta}$, and

$$
\begin{aligned}
& \mathscr{F}_{j}^{\delta}=\mathscr{F}^{+}\left(\alpha_{p, j}^{\delta,-}, u_{p, j}-\frac{\delta t}{\varrho_{p}} \nabla_{j}^{D} \Phi\right)+\mathscr{F}^{-}\left(\alpha_{p, j}^{\delta,+}, u_{p, j}-\frac{\delta t}{\varrho_{p}} \nabla_{j}^{D} \Phi\right), \\
& \alpha_{p, j}^{\delta, \pm}=(1-\delta) \alpha_{p, j}+\delta \alpha_{p, j \pm 1 / 2}
\end{aligned}
$$

for any $j=2, . ., J$. (We remind the reader the $\alpha_{p, j}$ is defined by the average (32).) A similar formula holds to define $\mathbb{D}^{(2) \delta}$. With these definitions at hand, we readily verify that

- $H(w, 1)=0$ is exactly equation $(37)$, 
- $\mathscr{F}_{j}^{0}=\alpha_{p, j} u_{p, j}-\frac{\delta t}{\varrho_{p}} \alpha_{p, j} \nabla_{j}^{D} \Phi$ so that $H(w, 0)=0$ becomes a linear equation where we recognize a standard 3 points approximation of the diffusion equation (28). Hence, $H(w, 0)=0$ admits a solution.

In order to apply Lemma B.1, we are left with the task of proving that

$$
\exists R>0 \text { such that } \forall(w, \delta) \in W \times[0 ; 1] \text {, if } \mathrm{H}(w, \delta)=0 \text { then }|w|_{W}<R .
$$

Let $w=\left(\Phi_{j+1 / 2}\right)_{j=1, . ., J} \in W$ and $\delta \in[0,1]$ such that $\mathrm{H}(w, \delta)=0$. We multiply the $(j+1 / 2)$-th component of this equation by $\delta x_{j+1 / 2} \Phi_{j+1 / 2}$ and sum over $j=2, . ., J$. We only detail the estimates for $\mathbb{D}^{(1)}$ but the same reasoning can be applied to $\mathbb{D}^{(2)}$. To start with, discrete integration by part yields

$$
\sum_{j=1}^{J} \delta x_{j+1 / 2} \frac{\mathscr{F}_{j+1}^{\delta}-\mathscr{F}_{j}^{\delta}}{\delta x_{j+1 / 2}} \Phi_{j+1 / 2}=-\sum_{j=2}^{J} \delta x_{j} \mathscr{F}_{j}^{\delta} \nabla_{j}^{D} \Phi .
$$

The key point is now to observe that, for $j=2, . ., J$,

$$
\mathscr{F}_{j}^{\delta}=\alpha_{p, j}^{\delta}\left(u_{p, j}-\frac{\delta t}{\varrho_{p}} \nabla_{j}^{D} \Phi\right)-\frac{1}{2}\left[\mathscr{F}|\cdot|\left(\alpha_{p, j}^{\delta,-}, u_{p, j}-\frac{\delta t}{\varrho_{p}} \nabla_{j}^{D} \Phi\right)-\mathscr{F}|\cdot|\left(\alpha_{p, j}^{\delta,+}, u_{p, j}-\frac{\delta t}{\varrho_{p}} \nabla_{j}^{D} \Phi\right)\right]
$$

where $\alpha_{p, j}^{\delta}=\left(\alpha_{p, j}^{\delta,-}+\alpha_{p, j}^{\delta,+}\right) / 2$ and $\mathscr{F}|\cdot|$ is defined by $\mathscr{F}^{\mid} \cdot \mid\left(\alpha_{p}, u_{p}\right)=\mathscr{F}^{+}\left(\alpha_{p}, u_{p}\right)-$ $\mathscr{F}^{-}\left(\alpha_{p}, u_{p}\right)$. The explicit expression $(29)-(30)$ of $\mathscr{F}^{+}$and $\mathscr{F}^{-}$leads to

$$
\mathscr{F}|\cdot|\left(\alpha_{p}, u_{p}\right)= \begin{cases}\alpha_{p}\left|u_{p}\right| & \text { if }\left|u_{p}\right| \geqslant c\left(\alpha_{p}\right) \\ \frac{\alpha_{p}}{2 c\left(\alpha_{p}\right)}\left(u_{p}{ }^{2}+c\left(\alpha_{p}\right)^{2}\right) & \text { if }\left|u_{p}\right| \leqslant c\left(\alpha_{p}\right)\end{cases}
$$

and we readily see that

$$
0 \leqslant \mathscr{F}^{|\cdot|}\left(\alpha_{p}, u_{p}\right) \leqslant \alpha_{p} \max \left(\left|u_{p}\right|, c\left(\alpha_{p}\right)\right) \leqslant \alpha_{p}\left(\left|u_{p}\right|+c\left(\alpha_{p}\right)\right)
$$

holds. It is convenient to set

$$
\varepsilon_{j}=\operatorname{sgn}\left(\nabla_{j}^{D} \Phi\right)
$$

and we shall use the notation $\alpha_{p, j}^{\delta, \varepsilon_{j}}$ accordingly. Since $\mathscr{F}|\cdot|\left(\alpha_{p}, u_{p}\right) \geqslant 0$, we can bound $\left(-\mathscr{F}_{j}^{\delta} \nabla_{j}^{D} \Phi\right)$ from below as follows

$$
-\mathscr{F}_{j}^{\delta} \nabla_{j}^{D} \Phi \geqslant \alpha_{p, j}^{\delta}\left(\frac{\delta t}{\varrho_{p}} \nabla_{j}^{D} \Phi-u_{p, j}\right) \nabla_{j}^{D} \Phi-\frac{1}{2} \mathscr{F}|\cdot|\left(\alpha_{p, j}^{\delta, \varepsilon_{j}}, u_{p, j}-\frac{\delta t}{\varrho_{p}} \nabla_{j}^{D} \Phi\right)\left|\nabla_{j}^{D} \Phi\right| .
$$

Then, applying the Young inequality we find, for all $\tau>0$,

$$
-\mathscr{F}_{j}^{\delta} \nabla_{j}^{D} \Phi \geqslant\left(1-\frac{\tau}{2}\right) \frac{\delta t}{\varrho_{p}} \alpha_{p, j}^{\delta}\left|\nabla_{j}^{D} \Phi\right|^{2}-\frac{1}{2 \tau} \frac{\varrho_{p}}{\delta t} \alpha_{p, j}^{\delta}\left|u_{p, j}\right|^{2}-\frac{1}{2} \mathscr{F}|\cdot|\left(\alpha_{p, j}^{\delta, \varepsilon_{j}}, u_{p, j}-\frac{\delta t}{\varrho_{p}} \nabla_{j}^{D} \Phi\right)\left|\nabla_{j}^{D} \Phi\right| .
$$

With (53) for $\mathscr{F}|\cdot|$ and the Young inequality, we obtain

$$
\begin{aligned}
\mathscr{F}\left|\cdot\left(\alpha_{p, j}^{\delta, \varepsilon_{j}}, u_{p, j}-\frac{\delta t}{\varrho_{p}} \nabla_{j}^{D} \Phi\right)\right| \nabla_{j}^{D} \Phi \mid & \leqslant \alpha_{p, j}^{\delta, \varepsilon_{j}}\left(\left|\frac{\delta t}{\varrho_{p}} \nabla_{j}^{D} \Phi-u_{p, j}\right|+c\left(\alpha_{p, j}^{\delta, \varepsilon_{j}}\right)\right)\left|\nabla_{j}^{D} \Phi\right| \\
& \leqslant\left(1+\frac{\tau}{2}\right) \frac{\delta t}{\varrho_{p}} \alpha_{p, j}^{\delta, \varepsilon_{j}}\left|\nabla_{j}^{D} \Phi\right|^{2}+\frac{1}{2 \tau} \frac{\varrho_{p}}{\delta t} \alpha_{p, j}^{\delta, \varepsilon_{j}}\left(\left|u_{p, j}\right|+c\left(\alpha_{p, j}^{\delta, \varepsilon_{j}}\right)\right)^{2} .
\end{aligned}
$$


Finally, we arrive at

$-\mathscr{F}_{j}^{\delta} \nabla_{j}^{D} \Phi \geqslant\left(2(2-\tau) \alpha_{p, j}^{\delta}-(2+\tau) \alpha_{p, j}^{\delta, \varepsilon_{j}}\right) \frac{\delta t}{4 \varrho_{p}}\left|\nabla_{j}^{D} \Phi\right|^{2}-\frac{1}{2 \tau} \frac{\varrho_{p}}{\delta t} \alpha_{p, j}^{\delta}\left|u_{p, j}\right|^{2}-\frac{1}{4 \tau} \frac{\varrho_{p}}{\delta t} \alpha_{p, j}^{\delta, \varepsilon_{j}}\left(\left|u_{p, j}\right|+c\left(\alpha_{p, j}^{\delta, \varepsilon_{j}}\right)\right)^{2}$.

However, going back to the definition of $\alpha_{p, j}^{\delta}$ and $\alpha_{p, j}^{\delta, \varepsilon_{j}}$ yields

$$
\begin{aligned}
2(2-\tau) \alpha_{p, j}^{\delta}-(2+\tau) \alpha_{p, j}^{\delta, \varepsilon_{j}} & =2 \alpha_{p, j}^{\delta,-\varepsilon_{j}}-\tau\left(\alpha_{p, j}^{\delta,-\varepsilon_{j}}+2 \alpha_{p, j}^{\delta, \varepsilon_{j}}\right) \\
& \geqslant 2 \min \left(\alpha_{p, j-1 / 2}, \alpha_{p, j+1 / 2}\right)-3 \tau \max \left(\alpha_{p, j-1 / 2}, \alpha_{p, j+1 / 2}\right) .
\end{aligned}
$$

Hence, we can take

$$
\tau=\frac{\min \left(\alpha_{p, j-1 / 2}, \alpha_{p, j+1 / 2}\right)}{3 \max \left(\alpha_{p, j-1 / 2}, \alpha_{p, j+1 / 2}\right)}>0
$$

and we obtain

$$
-\mathscr{F}_{j}^{\delta} \nabla_{j}^{D} \Phi \geqslant \min \left(\alpha_{p, j-1 / 2}, \alpha_{p, j+1 / 2}\right) \frac{\delta t}{4 \varrho_{p}}\left|\nabla_{j}^{D} \Phi\right|^{2}-\frac{1}{2 \tau} \frac{\varrho_{p}}{\delta t} \alpha_{p, j}^{\delta}\left|u_{p, j}\right|^{2}-\frac{1}{4 \tau} \frac{\varrho_{p}}{\delta t} \alpha_{p, j}^{\delta, \varepsilon_{j}}\left(\left|u_{p, j}\right|+c\left(\alpha_{p, j}^{\delta, \varepsilon_{j}}\right)\right)^{2} .
$$

We now sum over $j=2, . ., J$. Owing to (52) and bearing in mind the expression of $\tau$, we find

$$
\begin{aligned}
& \min _{i=1, . ., J}\left(\alpha_{p, i+1 / 2}\right) \frac{\delta t}{4 \varrho_{p}} \sum_{j=2}^{J} \delta x_{j}\left|\nabla_{j}^{D} \Phi\right|^{2} \\
& \leqslant \frac{3}{2} \frac{\varrho_{p}}{\delta t} \sum_{j=2}^{J} \delta x_{j+1 / 2} \frac{\left(\max \left(\alpha_{p, j-1 / 2}, \alpha_{p, j+1 / 2}\right)\right)^{2}}{\min \left(\alpha_{p, j-1 / 2}, \alpha_{p, j+1 / 2}\right)}\left[2\left|u_{p, j}\right|^{2}+\left(c\left(\max \left(\alpha_{p, j-1 / 2}, \alpha_{p, j+1 / 2}\right)\right)\right)^{2}\right] \\
& +\sum_{j=1}^{J} \delta x_{j}\left[\mathbb{D}^{(1) \delta}\left(\alpha_{p}, u_{p}-\frac{\delta t}{\varrho_{p}} \nabla^{D} \Phi\right)\right]_{j+1 / 2} \Phi_{j+1 / 2}
\end{aligned}
$$

Note that the first term of the right-hand-side is independent of $w$ and $\delta$. We apply the same reasoning for $\mathscr{G}_{j+1}^{\delta}$ and $\mathbb{D}^{(2) \delta}$. This leads to an inequality which is similar to (54). When summing the inequalities, bearing in mind $\mathrm{H}(w, \delta)=0$, the term

$$
\sum_{j=1}^{J} \delta x_{j}\left[\mathbb{D}^{(1) \delta}\left(\alpha_{p}, u_{p}-\frac{\delta t}{\varrho_{p}} \nabla^{D} \Phi\right)+\mathbb{D}^{(2) \delta}\left(\alpha_{f}, u_{f}-\frac{\delta t}{\varrho_{f}} \nabla^{D} \Phi\right)\right]_{j+1 / 2} \Phi_{j+1 / 2}
$$

in the right-hand-side vanishes and we get $|w|_{W}<R$ where $R$ does not depend on $w$ and $\delta$. The assumptions of Lemma B.1 are verified. We get the existence of a solution to the nonlinear problem (37).

\section{References}

[1] M. J. Andrews and P. J. O'Rourke. The multiphase particle-in-cell (MP-PIC) method for dense particulate flows. Int. J. Multiphase Flow, 22(2):379-402, 1996. 
[2] S. V. Apte, K. Mahesh, and T. Lundgren. A Eulerian-Lagrangian model to simulate two-phase/particulate flows. In Annual Research Briefs, Center for Turbulence Research, NASA \&S Stanford University, pages 161-171, 2003.

[3] F. M. Auzerais, R. Jackson, and W. B. Russel. The resolution of shocks and the effects of compressible sediments in transient settling. J. Fluid. Mech., 195:437$482,1988$.

[4] S. Benzoni-Gavage and D. Serre. Multidimensional hyperbolic partial differential equations: First-order systems and applications. Oxford Mathematical Monographs. Oxford Univ. Press, 2006.

[5] F. Berthelin, T. Goudon, and S. Minjeaud. Consistency analysis of a 1D finite volume scheme for barotropic Euler models. In Finite Volumes for Complex Applications VII; Methods, Theoretical Aspects, and Elliptic, Parabolic and Hyperbolic Problems, Berlin, volume 77 and 78 of Springer Proceedings in Mathematics 86 Statistics, pages 97-106. Springer, 2014.

[6] F. Berthelin, T. Goudon, and S. Minjeaud. Kinetic schemes on staggered grids for barotropic Euler models: entropy-stability analysis. Math. Comp., 2015. To appear.

[7] S. Boivin, F. Cayré, and J.-M. Hérard. Un schéma volumes finis pour la simulation d'écoulements diphasiques gaz particules à deux phases incompressibles sur maillage triangulaire. Revue Européenne des Eléments Finis, 10(5):539-574, 2001.

[8] F. Bouchut. Construction of BGK models with a family of kinetic entropies for a given system of conservation laws. J. Statist. Phys., 95(1-2):113-170, 1999.

[9] F. Bouchut. Nonlinear stability of finite volume methods for hyperbolic conservation laws and well-balanced schemes for sources. Frontiers in Math. Birkhauser, 2004.

[10] J. A. Carrillo and T. Goudon. Stability and asymptotic analysis of a fluid-particle interaction model. Comm. PDE., 31(9):1349-1379, 2006.

[11] J. A. Carrillo, T. Goudon, and P. Lafitte. Simulation of fluid \& particles flows: Asymptotic preserving schemes for bubbling and flowing regimes. J. Comput. Phys., 227(16):7929-7951, 2008.

[12] A. Chorin. The numerical solution of the Navier-Stokes equations for an incompressible fluid. Bull. Amer. Math. Soc., 73:pp. 928-931, 1967.

[13] A. Chorin. Numerical solution of the Navier-Stokes equations. Math. Comp., 22(104):745-762, 1968.

[14] A. Chorin. On the convergence of discrete approximations to the Navier-Stokes equations. Math. Comp., 23(106):341-353, 1969.

[15] K. Chueh, C. Conley, and J. Smoller. Positively invariant regions for systems of nonlinear equations. Indiana. U. Math. J., 26:373-392, 1977.

[16] F. Clarelli, C. Di Russo, N. Natalini, and M. Ribot. Mathematical models for biofilms on the surface of monuments. In Applied and Industrial Mathematics in Italy III, 9th Conference SIMAI, volume 82 of Advances in Mathematics for Applied Sciences. World Scientific, 2009. 
[17] F. Clarelli, C. Di Russo, N. Natalini, and M. Ribot. A fluid dynamics model of the growth of phototrophic biofilms. J. Math. Biology, 66(7):1387-408, 2013.

[18] R. Clift, J. Grace, and M. Weber. Bubbles, drops and particles. Academic Press, 1978.

[19] F. Coron and B. Perthame. Numerical passage from kinetic to fluid equations. SIAM J. Numer. Anal., 28:26-42, 1991.

[20] K. Deimling. Nonlinear functional analyis. Dover Books on Mathematics. Dover, 2010 .

[21] O. Desjardins, R.O. Fox, and P. Villedieu. A quadrature-based moment method for dilute fluid-particle flows. J. Comput. Phys., 227:2514-2539, 2008.

[22] A Faghri and Y. Zhang. Transport Phenomena in Multiphase Systems. Academic Press, 2006.

[23] T. Goudon, P.-E. Jabin, and A. Vasseur. Hydrodynamic limit for the VlasovNavier-Stokes equations. I. Light particles regime. Indiana Univ. Math. J., 53(6):1495-1515, 2004.

[24] T. Goudon, P.-E. Jabin, and A. Vasseur. Hydrodynamic limit for the VlasovNavier-Stokes equations. II. Fine particles regime. Indiana Univ. Math. J., 53(6):1517-1536, 2004.

[25] T. Goudon, S. Jin, and B. Yan. Simulation of fluid-particles flows: heavy particles, flowing regime and asymptotic-preserving schemes. Comm. Math. Sci., 10(1):355$385,2012$.

[26] T. Goudon, J.-G. Liu, S. Jin, and B. Yan. Asymptotic-preserving schemes for kinetic-fluid modeling of disperse two-phase flows. J. Comput. Phys., 246:145164, 2013.

[27] T. Goudon, J.-G. Liu, S. Jin, and B. Yan. Asymptotic-preserving schemes for kinetic-fluid modeling of disperse two-phase flows with variable fluid density. Int. J. Numerical Methods in Fluids, 75(2):81-102, 2014.

[28] J. L. Guermond, P. Minev, and J. Shen. An overview of projection methods for incompressible flows. Comput. Methods Appl. Mech. Engrg., 195(44-47):6011$6045,2006$.

[29] S. E. Harris and D. G. Crighton. Solitons, solitary waves, and voidage disturbances in gas-fluidized beds. J. Fluid. Mech., 266:243-276, 1994.

[30] R. Herbin, W. Kheriji, and J.-C. Latche. Staggered schemes for all speed flows. ESAIM: ProcS, 35:122-150, 2012.

[31] S. Jin and X. Li. Multi-phase computations of the semiclassical limit of the Schrödinger equation and related problems: Whitham vs. Wigner. Physica D, 182:46-85, 2003.

[32] C.K.K. Lun and S.B. Savage. Kinetic theory for inertia flows of dilute turbulent gas-solids mixtures. In T. Pöschel and N. V Brilliantov, editors, Granular Gas Dynamics, volume 624 of Lect. Notes Phys., pages 267-289. Springer, 2003. 
[33] P. J. O'Rourke. Collective drop effects on vaporizing liquid sprays. $\mathrm{PhD}$ thesis, Princeton University, NJ, 1981. Available as Technical Report \#87545 Los Alamos National Laboratory.

[34] N. A. Patankar and D. D. Joseph. Lagrangian numerical simulation of particulate flows. Int. J. Multiphase Flow, 27:1685-1706, 2001.

[35] N. A. Patankar and D. D. Joseph. Modeling and numerical simulation of particulate flows by the Eulerian-Lagrangian approach. Int. J. Multiphase Flow, 27:1659-1684, 2001.

[36] B. Perthame. Second order Boltzmann schemes for compressible Euler equations in one and two space dimension. SIAM J. Numer. Anal., 29(1):1-19, 1992.

[37] B. Perthame. Kinetic formulation of conservation laws. Oxford Lecture Series in Math. and its Appl. Oxford University Press, 2003.

[38] E. B. Pitman and L. Le. A two-fluid model for avalanche and debris flows. Phil. Trans. R. Soc. A, 363:1573-1601, 2005.

[39] A. Prosperetti and G. Tryggvason. Computational Methods for Multiphase Flows. Cambridge Univ. Press, 2007.

[40] D. Serre. Systems of Conservation Laws, II: Geometric structures, oscillations and initial-boundary-value problems. Cambridge Univ. Press, 2000.

[41] D. M. Snider, P. J. O'Rourke, and M. J. Andrews. Sediment flow in inclined vessels calculated using a multiphase particle-in-cell model for dense particle flows. Int. J. of Multiphase Flow, 24:1359-1382, 1998.

[42] R. Temam. Sur l'approximation de la solution des équations de Navier-Stokes par la méthode des pas fractionnaires II. Arch. Rational Mech. Anal., 33:377-385, 1969. 